\title{
Joint Power and Time Allocation in Full-Duplex Wireless Powered Communication Networks
}

\author{
Yongbo Cheng, ${ }^{1,2}$ Pengcheng Fu, ${ }^{1,2}$ Yuchao Chang, ${ }^{1,2}$ Baoqing Li, ${ }^{1}$ and Xiaobing Yuan ${ }^{1}$ \\ ${ }^{1}$ Science and Technology on Microsystem Laboratory, Shanghai Institute of Microsystem and Information Technology, \\ Chinese Academy of Sciences, Shanghai 200050, China \\ ${ }^{2}$ University of Chinese Academy of Sciences, Beijing 100049, China \\ Correspondence should be addressed to Yongbo Cheng; whcyb@mail.ustc.edu.cn
}

Received 26 May 2016; Revised 21 July 2016; Accepted 31 July 2016

Academic Editor: Josip Lorincz

Copyright (C) 2016 Yongbo Cheng et al. This is an open access article distributed under the Creative Commons Attribution License, which permits unrestricted use, distribution, and reproduction in any medium, provided the original work is properly cited.

\begin{abstract}
We consider a full-duplex wireless powered communication network (WPCN) with one hybrid access point (H-AP) and a set of distributed users, where downlink wireless energy broadcasting is employed at H-AP and at the same time, uplink wireless information transmission takes place at users in a time-division multiple access manner. We extend proportional fair scheduler to this category of network when dealing with "doubly near-far problem," where users far away from H-AP achieve low throughput but suffer from both low harvested energy and high data transmission power consumption. We jointly optimize power and time allocation for each user to achieve proportional fairness while controlling the energy consumption offset for network to a low level. By using optimization techniques, the optimal transmit power and transmission time for users are obtained via proposed algorithm. Simulation results confirm the positive effect on improving the fairness metric and reducing energy consumption offset for network.
\end{abstract}

\section{Introduction}

Recently, there has been upsurge of research interests in RF(Radio Frequency-) enabled wireless energy transfer (WET) technology, which is a promising solution to extend lifetime in energy-constrained wireless networks, such as wireless sensor networks and cellular networks [1-3]. As one important application of RF-enabled WET, wireless powered communication network (WPCN) is a category of network where wireless devices are utilizing the energy harvested from RF signal to transmit/decode information to/from other devices [4]. Since wireless devices are usually equipped with battery, which may be inconvenient or difficult to recharge after being deployed, the communication network has a limited operational lifetime. To prolong the lifetime of these devices, energy harvesting is utilized to provide a feasible power $\mathrm{s}$ upply. Ambient energy sources for energy harvesting including solar, motion, wind, thermoelectric effects, and fuel are adopted in wireless communication networks $[5,6]$, which may pose uncertainty in the amount of harvested energy [7]. Nevertheless, dedicated power transmitter is applied to meet the demand of quality constrained applications in wireless powered communication networks.

A promising research area in WPCN is wireless energy transfer in the downlink (DL) and information transfer in the uplink (UL) via an energy/information full-duplex (FD) approach [8]. FD communications have gained a great deal of interest with transmission and reception simultaneously on the same frequency, which has the potential to double the spectral efficiency [9-11]. In practice, self-interference cancellation (SIC) techniques in propagation-domain, analogcircuit-domain, and digital-domain [10] have made fullduplex communication more practical [12]. In contrast to conventional half-duplex transmission, FD is adopted in WPCN $[7,13-16]$ to enhance the system performance since RF signals can carry both of energy and information. In [7], the authors proposed FD medium access control (MAC) protocol using hybrid access point (H-AP) with enabling simultaneous downlink wireless power transfer and uplink wireless information transfer. In [13], FD is utilized in WPCN with energy broadcasting in DL and information transmission in UL simultaneously. 
A practical challenge named "doubly near-far problem" is emphasized in WPCN [17], where users far away from the transmitter achieve very low throughput, as they suffer from both low harvested energy in DL and high transmission power consumption in UL $[3,4,8,17,18]$. This problem induces unfairness among users that are far away from the H-AP. The authors in $[7,13,14]$ considered the resource allocation among users to maximize the sum-throughput over UL in WPCN, which is resource efficient but also can be unfair among users with resource sharing. To tackle this phenomenon, the authors in [17] proposed a new performance metric named common-throughput, which enables users to have an equal rate allocation regardless of their distance. Furthermore, proportional fair scheduler can be implemented to tackle this problem, which will bring a tradeoff between fairness and throughput [19-21].

In this paper, we adopt FD technique to the area of WPCN, with H-AP broadcasting wireless energy in DL and at the same time user transmitting information via timedivision medium access (TDMA) manner in UL. We apply proportional fair scheduler over the FD WPCN to maximize utility function for fairness, while considering minimizing energy consumption offset for network, with optimally allocating transmit power and time for users.

The main contributions of this paper are listed as follows:

(i) We propose a FD WPCN model, which enables simultaneous wireless energy transfer in DL at $\mathrm{H}-$ AP and wireless information transfer in UL for multiusers. We also propose a time-division based MAC protocol, which can avoid conflict for multiusers when transmitting their independent information in UL.

(ii) To tackle the interesting "doubly near-far problem" in FD WPCN, we characterize a fundamental optimization problem by jointly optimizing the power and time allocation for users, with maximizing the bandwidth utility function for proportional fairness, as well as minimizing the energy consumption offset for network.

(iii) For the optimization problem, we utilize the optimization techniques to obtain the optimal power and time allocation. Furthermore, we propose an algorithm to achieve proportional fairness and energy efficiency in FD WPCN.

(iv) We investigate the impact of number of users and transmit power of energy broadcasting on performance via simulation. Simulation results verify that the proposed algorithm enhances the fairness index and reduces energy consumption offset for network.

The rest of our paper is organized as follows: system model and proportional fairness are introduced in Section 2. Moreover, we introduce a time-division based MAC protocol for FD WPCN in Section 3. A general problem formulation is given in Section 4. In addition, our proposed algorithm is discussed in Section 5. After simulation, the numerical results and discussions are shown in Section 6. We conclude this paper in Section 7.

\section{System Model and Proportional Fairness}

In this section, we establish the models of data transmission rate, energy harvesting and consumption, and proportional fairness in FD WPCN. Some notations are listed in Notation.

We consider WPCN with a dual-functional H-AP expressed as $s$ and $M$ users denoted by $u_{i}, i \in[1, M]$. The set of all users is expressed as $U$, with cardinality $M$. H-AP acts as not only an access point to collect data from users but also an energy transmitter that broadcasts wireless energy. A H-AP transmits both the energy and information to/from the subscribers [3]. Specifically, we assume that $s$ has a high capacity battery and $u_{i}$ has a finite capacity battery to store the harvested wireless energy. The battery in $u_{i}$ is fully charged initially in a practical causal energy system $[7,13,22]$, which represents that the energy of future RF signal is not available at the current time slot [22]. In addition, $u_{i}$ is assumed to have a stable transmit power for data transmission and the amount of energy in battery decreases with time running.

As shown in Figure 1, we assume that WPCN can operate on two phases: full-duplex (FD) phase and nonFD phase. FD phase is utilized for wireless energy charging and data transmission simultaneously to enhance network performance, and non-FD phase is mainly used for network structure formation with message transmission. In FD phase, we assume that $s$ and $M$ can operate on the same frequency band (in-band) with full-duplex antennas [14, 23]. H-AP $s$ broadcasts wireless energy in the DL and at the same time, user $u_{i}$ transmits information in the UL via time-division based MAC protocol. In addition, we assume that $s$ and $u_{i}$ have SIC ability. Moreover, it should be pointed out that the full-duplex in this paper is different from conventional FD communication, where FD devices are only for information transmission [7]. Conventional FD communication represents that information transmission and reception take place in the same frequency band and the antenna [3]. By contrast, our FD term refers to energy broadcasting/harvesting as well as data reception/transmission at the same time. In Figure 1(a), in FD phase, $g_{i}$ represents the DL channel power gain for energy broadcasting from $s$ to $u_{i} . h_{U, i}$ represents the UL channel power gain for data transmission from $u_{i}$ to $s$. As Figure 1(b) shows, in non-FD phase, data transmission is utilized by H-AP to send admission control results to decide which incoming users can be accepted into the network [24]. $h_{D, i}$ represents the channel power gain in DL for data transmission. In addition, since $s$ can send messages back to $u_{i}$, data reception acknowledgment can also be used in this phase to enhance the performance of MAC protocol.

Figure 2 shows the transceiver structure of H-AP $s$ and user $u_{i} . s$ and $u_{i}$ are equipped with a single antenna [14] using a circulator as duplexer [25] to enable FD wireless energy broadcasting/harvesting and information reception/transmission. In H-AP, battery provides energy for information transceiver and energy transmitter. Furthermore, energy harvester in $u_{i}$ collects wireless energy to recharge the battery, which can provide energy for the information transceiver. The transceiver structure can operate on two phases as we have assumed. In FD phase, as shown in Figure 2(a), the information transceiver in H-AP works in 


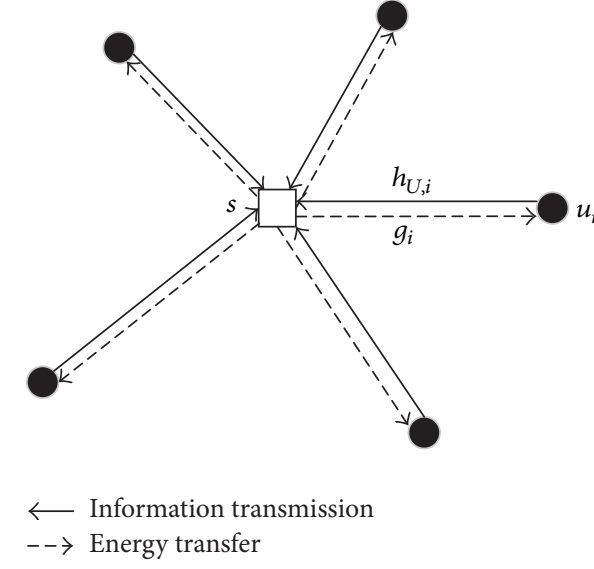

(a)

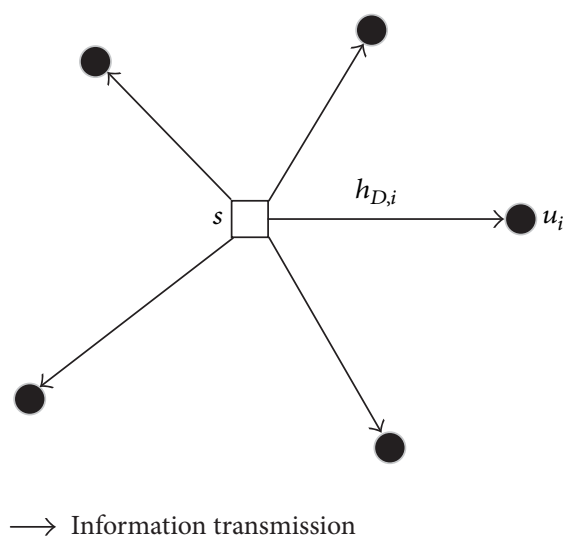

(b)

FIGURE 1: System model. (a) WPCN in FD phase; (b) WPCN in non-FD phase.

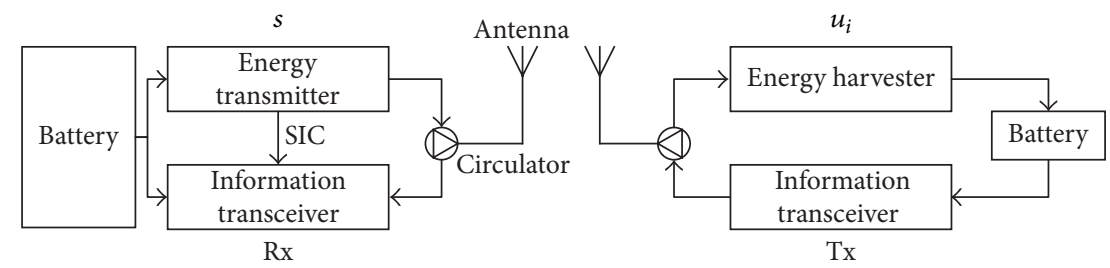

(a)

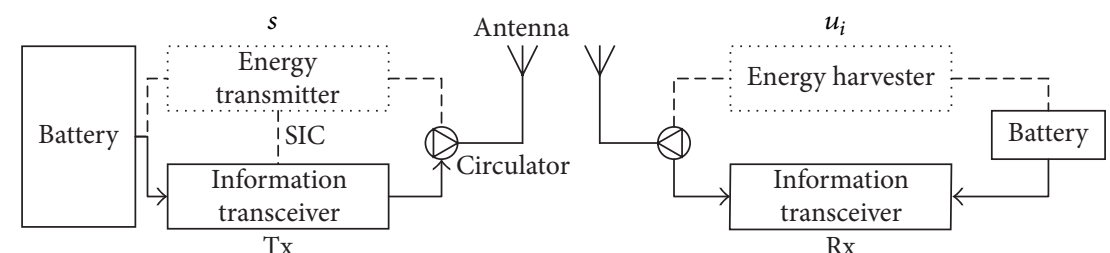

(b)

FIgURE 2: Transceiver structure of H-AP $s$ and user $u_{i}$. (a) WPCN in FD phase; (b) WPCN in non-FD phase.

Rx state to decode the information from $u_{i}$, while the state of information transceiver in $u_{i}$ is Tx to send information in UL. Moreover, self-interference canceller is adopted in $\mathrm{H}$-AP to cancel the interference from energy signals when information transceiver receives information signals in UL. Energy transmitter in H-AP broadcasts energy in DL with user $u_{i}$ harvesting the wireless energy and at the same time, $u_{i}$ sends data in UL with H-AP decoding the information. As shown in Figure 2(b), in non-FD phase, the energy transmitter in H-AP and energy harvester in user $u_{i}$ stop to work in order to avoid interfering the transmission of information. The information transceiver in H-AP works in Tx state, while the information transceiver in user $u_{i}$ operates in $\mathrm{Rx}$ state. Information can be transmitted in this wireless link as Figure 1(b) shows.

As Figure 3(a) shows, $s$ and $u_{i}$ operate in FD phase. $P_{B}$ is the transmit power of energy broadcasting in DL at $s$. $P_{U, i}$ is the transmit power for data transmission in UL at $u_{i}$, with $P_{U, i} \leq P_{U, \max }$, and $P_{U, \max }$ is the maximum UL transmit power for data transmission. Moreover, $S_{B}$ is the equivalent SIC power gain at $s$ for energy broadcasting. $S_{D}$ denotes the equivalent power gain after SIC at $u_{i}$. It should be pointed out that $g_{i}$ and $h_{U, i}$ are distance-related terms, which can be denoted as $g_{i} \propto d_{i}^{-\alpha}$ and $h_{U, i} \propto d_{i}{ }^{-\alpha}$, where $d_{i}$ is the distance between $s$ and $u_{i}$ and $\alpha$ is the path loss exponent. In Figure $3(\mathrm{~b}), s$ and $u_{i}$ work in non-FD phase. The DL transmit power for data transmission at $s$ is denoted as $P_{D, s}$. Similar to $h_{U, i}, h_{D, i} \propto d_{i}^{-\alpha}$.

2.1. Data Transmission Rate. Furthermore, in Figure 3(a), the received power from $u_{i}$ to $s$ for data transmission at $s$ is written by $P_{U, s}=h_{U, i} P_{U, i}$. As a result, the instantaneous UL data transmission rate $R_{U, i}$ can be expressed as $[7,13]$

$$
R_{U, i}=\ln \left(1+\frac{P_{U, s}}{P_{B} S_{B}+\sigma^{2}}\right)=\ln \left(1+\frac{h_{U, i} P_{U, i}}{P_{B} S_{B}+\sigma^{2}}\right),
$$




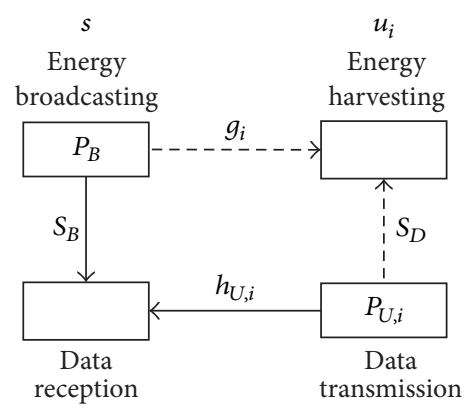

(a)

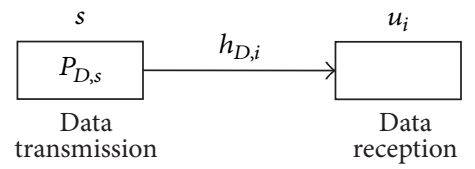

(b)

FIGURE 3: (a) Wireless energy transfer and data transmission model in FD phase. (b) Data transmission model in non-FD phase.

where $\sigma^{2}$ is the noise power at $u_{i}$. Moreover, as shown in Figure 3(b), the received power at $u_{i}$ can be expressed as $P_{D, i}=h_{D, i} P_{D, s}$. The instantaneous DL data transmission rate $R_{D, i}$ can be denoted by

$$
R_{D, i}=\ln \left(1+\frac{P_{D, i}}{\sigma^{2}}\right)=\ln \left(1+\frac{h_{D, i} P_{D, s}}{\sigma^{2}}\right) .
$$

We also assume that $f_{U, i}$ and $f_{D, i}$ are the flow rates from $u_{i}$ to $s$ in UL and $s$ to $u_{i}$ in DL, respectively. Naturally, we have

$$
\begin{aligned}
& f_{U, i} \leq R_{U, i}, \\
& f_{D, i} \leq R_{D, i} .
\end{aligned}
$$

2.2. Energy Harvesting and Energy Consumption. For energy harvesting, $u_{i}$ harvests energy in FD phase as Figure 3(a) shows. The amount of power harvested in $u_{i}$ can be expressed as

$$
P_{H, i}=\eta_{i} g_{i} P_{B}+\eta_{D} S_{D} P_{U, i},
$$

where $\eta_{i}$ is the energy harvesting efficiency at $u_{i}, \eta_{i} \in(0,1) . \eta_{D}$ is the power transfer efficiency at $u_{i}, \eta_{D} \in(0,1)$. Specifically, the first part in (4) represents the power harvested from $s$, and the second part is the power from itself for SIC.

For energy consumption of data transmission, the most power hungry block is radio transceiver. This consumption of energy can be decomposed into two parts: transmitting energy consumption and reception consumption [26]. At $u_{i}$, the rate of energy consumption for transmitting one unit data flow in UL from $u_{i}$ to $s$ is denoted by $C_{U, i}$. Meanwhile, at $s$, the rate of reception energy consumption for one data flow in UL from $u_{i}$ to $s$ is expressed as $\rho_{U, i}$, which is a receiver-related constant term. Similarly, $C_{D, i}$ and $\rho_{D, i}$ are the rate of transmitting and receiving energy consumption for one unit DL data flow, respectively. Then, the rate of energy consumption for data flow from $u_{i}$ to $s$ in UL and $s$ to $u_{i}$ in DL is expressed as $p_{D, i}$ and $p_{U, i}$, respectively. They can be written by

$$
\begin{aligned}
& p_{D, i}=\left(C_{D, i}+\rho_{D, i}\right) f_{D, i}, \\
& p_{U, i}=\left(C_{U, i}+\rho_{U, i}\right) f_{U, i},
\end{aligned}
$$

where $\beta_{1}$ is a transmitter-related constant term and $\beta_{2}$ is an amplifier-related coefficient term. Specifically, $C_{D, i}$ and $C_{U, i}$ are modified from [26] as

$$
\begin{aligned}
& C_{D, i}=\beta_{1} P_{D, s}+\beta_{2} d_{i}^{\alpha}, \\
& C_{U, i}=\beta_{1} P_{U, i}+\beta_{2} d_{i}^{\alpha} .
\end{aligned}
$$

2.3. Proportional Fairness. Proportional fairness is widely adopted to effectively balance user fairness and network performance (i.e., sum of bandwidth utilities of all users) [27, 28]. A fairness criterion was proposed by Kelly in [27]. Furthermore, a succinct definition of proportional fairness was described in [29]. According to [27], a bandwidth allocation with $M$ users, which is denoted by $\left\{b_{1}, b_{2}, \ldots, b_{M}\right\}$, is proportionally fair if it is feasible and if for any other feasible allocation $\left\{b_{1}{ }^{*}, b_{2}{ }^{*}, \ldots, b_{M}{ }^{*}\right\}$, the sum of differences between $\left\{b_{1}{ }^{*}, b_{2}{ }^{*}, \ldots, b_{M}{ }^{*}\right\}$ and $\left\{b_{1}, b_{2}, \ldots, b_{M}\right\}$ is either zero or negative $[29,30]$.

In this paper, we aim at constructing an assignment of user UL bandwidth in a proportional fair manner, which can bring a tradeoff between fairness and throughput (sum of bandwidth). The objective for proportional fairness is defined as weighted sum of the logarithm UL bandwidth [28, 29], which can be expressed as

$$
\max \sum_{i=1}^{M} \omega_{i} \log b_{i},
$$

where $\omega_{i}$ is the weight of user $u_{i}, 0 \leq \omega_{i} \leq 1$, and $\sum_{i=1}^{M} \omega_{i}=1$. $b_{i}$ is the UL bandwidth for $u_{i}$. Specifically, the sum inside the logarithm function is greater than zero; that is, $b_{i}>0$.

To measure fairness level, we adopt Jain's fairness index as the fairness metric, which is denoted by J. It was first proposed by Jain et al. in [31] as a quantitative index. It can provide guidance for the study of fairness in various issues. Jain's fairness index is set between 0 and 1. The larger Jain's fairness index, the better the fairness; that is, the fairness index is 1 , and the bandwidth allocation is $100 \%$ fair. The definition of Jain's fairness index for UL data transmission bandwidth in this paper is

$$
J=\frac{\left(\sum_{i=1}^{M} b_{i}\right)^{2}}{M \sum_{i=1}^{M} b_{i}^{2}} .
$$




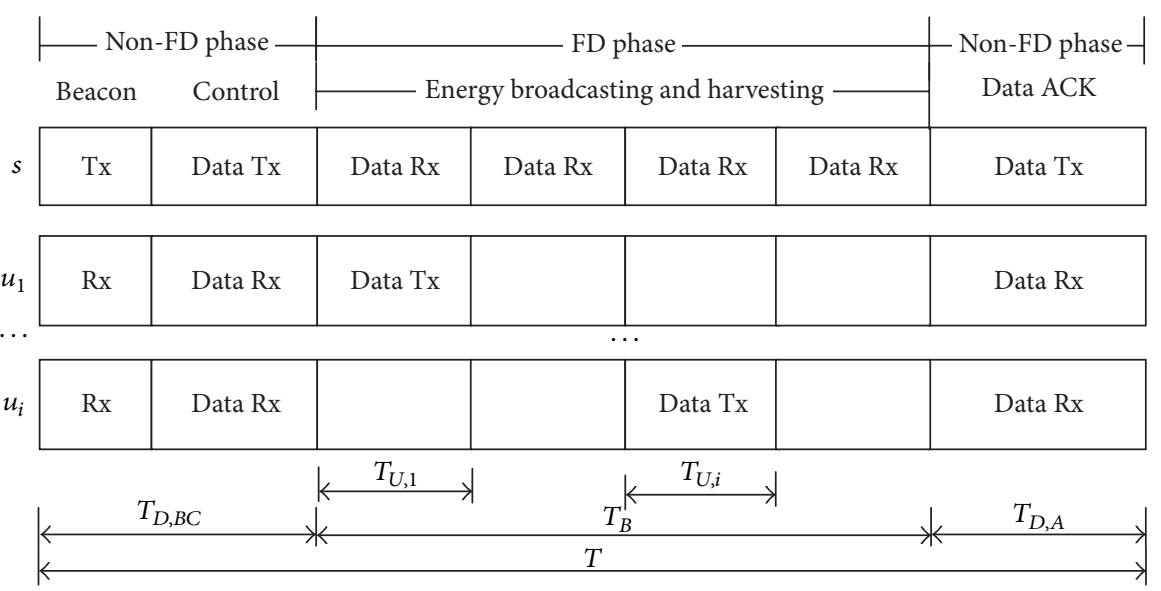

FIGURE 4: Full-duplex time-division based MAC protocol (FDTD-MAC).

\section{Time-Division Based MAC Protocol}

In this section, we introduce a contention-free FD timedivision based MAC protocol for WPCN (FDTD-MAC). The protocol can be divided into two stages: Network Formation Stage and Simultaneously Transfer Stage.

In Network Formation Stage, a cluster will be formed by protocol in star topology as described in Figure 1. $s$ transmits beacon frame periodically. $u_{i}$ remains reception state until it receives the beacon frame. After receiving the frame, $u_{i}$ will begin its timer to start an association request to $s$. In addition, $s$ can transmit control frame to $u_{i}$ for managing the access way of $u_{i}$. Before sending their requests frame, multiusers utilize a time-division based mechanism to avoid conflict. Moreover, an acknowledgment (ACK) frame will be returned to $u_{i}$ after $s$ receives the request frame. Specifically, $u_{i}$ with a larger received signal strength than a certain threshold can be able to join to $s$. Finally, the cluster will be formed.

In Simultaneously Transfer Stage, it provides the advantage of wireless information and energy transfer concurrently. $s$ broadcasts wireless energy in the downlink and $u_{i}$ uploads information in the uplink using time-division protocol simultaneously, which can avoid conflict at $s$ for receiving data from multiusers.

Figure 4 shows how the FDTD-MAC protocol works. First, it is in non-FD phase. $s$ sends beacon frame for network formation and network synchronization. Then, $s$ sends control or management frame to $u_{i}$. The channel state information and resource allocation results (e.g., time slot allocation information) can be transmitted through this kind of frame. Furthermore, the transmission time for beacon frame and control frame is denoted as $T_{D, B C}$. Second, it changes into FD phase. $s$ broadcasts energy in $T_{B}$, where $T_{B}=$ $\sum_{i=1}^{M} T_{U, i}$, and at the same time, $u_{i}$ uploads data to $s$ via timedivision medium access protocol to avoid conflict. $u_{i}$ 's data transmission time is expressed as $T_{U, i}$. $T_{U, i}$ is decided by the time allocation results at $s$ and is larger than the least required time duration for data transmission $T_{n}$ (i.e., $T_{U, i} \geq T_{n}$ ). Finally, after receiving the data from $u_{i}, s$ sends an ACK frame back to $u_{i}$. Moreover, the duration for data ACK transmission is denoted by $T_{D, A}$. The period of the protocol is expressed as $T$.

\section{Problem Formulation}

In this section, we first set up the fundamental energy consumption offset equations for entire network and $u_{i}$. Based on this equations, the "doubly near-far problem" in FD WPCN is explained in detail. To overcome this near-far problem, we address the proportional fairness among users with considering minimizing energy consumption offset, which is the amount of energy consumption minus energy harvesting. Finally, we formulate an optimization problem as system problem definition, which can be shown later.

\subsection{Energy Consumption Offset Equation}

4.1.1. Energy Consumption Offset Equation for Network. The total energy consumption of network can be expressed as

$$
E_{c}=E_{\mathrm{cal}}+E_{D}+E_{U}+E_{B}+E_{\mathrm{SIC}},
$$

where $E_{\text {cal }}$ is the total energy consumption for calculation at $s$ and $u_{i}, E_{D}$ is the total energy consumption for data transmission in DL from $s$ to $u_{i}$, and $E_{U}$ is the entire data transmission energy consumption in UL from $u_{i}$ to $s$. Moreover, $E_{B}$ is the energy consumption for energy broadcasting, and $E_{\text {SIC }}$ is the energy consumption for SIC at $s$ and $u_{i}$. Specifically, we define each part of energy consumption in (9) as

$$
\begin{aligned}
E_{\mathrm{cal}} & =E_{\mathrm{cal}, s}+\sum_{i=1}^{M} E_{\mathrm{cal}, i}, \\
E_{D} & =\sum_{i=1}^{M}\left(T_{D, B C}+T_{D, A}\right) p_{D, i}, \\
E_{U} & =\sum_{i=1}^{M} T_{U, i} p_{U, i},
\end{aligned}
$$




$$
\begin{aligned}
E_{B} & =T_{B} P_{B} \\
E_{\mathrm{SIC}} & =E_{\mathrm{SIC}, s}+\sum_{i=1}^{M} E_{\mathrm{SIC}, i} \\
& =\phi_{\mathrm{SIC}, s} P_{B} T_{B}+\sum_{i=1}^{M} \phi_{\mathrm{SIC}, i} P_{U, i} T_{U, i}
\end{aligned}
$$

where $E_{\mathrm{cal}, s}$ is the energy consumption for calculation at $s$ and $E_{\mathrm{cal}, i}$ is that at $u_{i}$. Moreover, $\phi_{\mathrm{SIC}, s}$ and $\phi_{\mathrm{SIC}, i}$ are the energy consumption coefficient term for SIC at $s$ and $u_{i}$.

The total harvested energy for network can be expressed as

$$
E_{H}=\sum_{i=1}^{M} T_{B} P_{H, i}
$$

Finally, the induced energy consumption offset for network in total is denoted by

$$
\begin{aligned}
E_{\mathrm{O}, N}= & E_{c}-E_{H} \\
= & E_{\mathrm{cal}, s}+\sum_{i=1}^{M} E_{\mathrm{cal}, i} \\
& +\sum_{i=1}^{M}\left(T_{D, B C}+T_{D, A}\right)\left(\beta_{1} P_{D, s}+\beta_{2} d_{i}^{\alpha}+\rho_{D, i}\right) R_{D, i} \\
& +\sum_{i=1}^{M} T_{U, i}\left(\beta_{1} P_{U, i}+\beta_{2} d_{i}^{\alpha}+\rho_{U, i}\right) R_{U, i} \\
& +\left(\sum_{i=1}^{M} T_{U, i}\right) P_{B}+\phi_{\mathrm{SIC}, s} P_{B}\left(\sum_{i=1}^{M} T_{U, i}\right) \\
& +\sum_{i=1}^{M} \phi_{\mathrm{SIC}, i} P_{U, i} T_{U, i} \\
& -\sum_{i=1}^{M}\left(\sum_{i=1}^{M} T_{U, i}\right)\left(\eta_{i} g_{i} P_{B}+\eta_{D} S_{D} P_{U, i}\right)
\end{aligned}
$$

Specifically, we notice that $E_{O, N}$ is related to transmit power $\mathbf{P} \triangleq\left\{P_{U, i}\right\}$ and time $\mathbf{T} \triangleq\left\{T_{U, i}\right\}$. With data flow $f_{D, i}=$ $R_{D, i}, f_{U, i}=R_{U, i}$, we can express it as $E_{O, N}(\mathbf{P}, \mathbf{T})$.

4.1.2. Energy Consumption Offset Equation for $u_{i}$. The energy consumption of $u_{i}$ can be expressed as

$$
E_{c, i}=E_{\mathrm{cal}, i}+E_{D, i}+E_{U, i}+E_{\mathrm{SIC}, i}
$$

where $E_{D, i}$ and $E_{U, i}$ are the energy consumption for data transmission at $u_{i}$ in DL and $\mathrm{UL}$, respectively. We can express them as

$$
\begin{aligned}
& E_{D, i}=\left(T_{D, B C}+T_{D, A}\right) \rho_{D, i} R_{D, i}, \\
& E_{U, i}=T_{U, i} p_{U, i} .
\end{aligned}
$$

In addition, the harvested energy for $u_{i}$ can be denoted by

$$
E_{H, i}=T_{B} P_{H, i}=T_{B}\left(\eta_{i} g_{i} P_{B}+\eta_{D} S_{D} P_{U, i}\right) .
$$

Finally, the induced energy consumption offset for $u_{i}$ can be expressed as

$$
\begin{aligned}
E_{\mathrm{O}, i}= & E_{c, i}-E_{H, i} \\
= & E_{\mathrm{cal}, i}+\left(T_{D, B C}+T_{D, A}\right) \rho_{D, i} R_{D, i} \\
& +T_{U, i}\left(\beta_{1} P_{U, i}+\beta_{2} d_{i}^{\alpha}+\rho_{U, i}\right) R_{U, i}+E_{\mathrm{SIC}, i} \\
& -\left(\sum_{i=1}^{M} T_{U, i}\right)\left(\eta_{i} g_{i} P_{B}+\eta_{D} S_{D} P_{U, i}\right) .
\end{aligned}
$$

4.2. Doubly Near-Far Problem in FD WPCN. A doubly nearfar problem in FD WPCN, which results in unfairness among users, is characterized in this part in detail. It represents that a user located closer to H-AP harvests more amount of wireless energy than a farther user but requires less power and less energy consumption for achieving the same information rate [17]. It also represents that the users far away from $\mathrm{H}$ AP achieve lower data rates than those located near H-AP with same transmit power. This near-far problem can reduce fairness among users that are distributed apart from the H-AP (with fixed time slot allocation) [3].

Figures 5 and 6 show the doubly near-far problem, with $\alpha=3,1 \leq d_{i} \leq 5(\mathrm{~m})$. We use this distance interval for the reason that if distance is less than $1 \mathrm{~m}$, it can be extreme unfairness among users, and if distance is greater than $5 \mathrm{~m}$, it does not make sense to evaluate it because the amount of harvested energy is very small. In practical implementation such as Powercast RF energy harvester, it has been introduced that the power harvested from RF signals operating at $915 \mathrm{MHz}$ at distance of $0.6 \mathrm{~m}$ and $11 \mathrm{~m}$ is $3.5 \mathrm{~mW}$ and $1 \mu \mathrm{W}$, respectively $[17,32]$. From Figure 5 , it can be observed that harvested energy level is very low at $u_{i}$ that is far away from H-AP, but transmit power level is high for achieving the same transmission rate. It undoubtedly causes unfairness among users that located at different distance from $\mathrm{H}$-AP. This phenomenon can be explained as the equations show. From (15), we can observe that $E_{H, i}$ decreases with $d_{i}$ increasing for given $P_{B}$, with $g_{i} \propto d_{i}^{-\alpha}$. In addition, it is also observed that $E_{H, i}$ decreases with $P_{U, i}$ decreasing. Since the value of $\eta_{D} S_{D}$ is very small, $P_{U, i}$ has a negligible effect on the result of harvested energy. From (1), we can observe that $R_{U, i}$ increases with $d_{i}$ decreasing for given $P_{B}$, with $h_{U, i} \propto$ $d_{i}{ }^{-\alpha}$. In others words, for achieving the same UL information transmission rate $R_{U, i}, P_{U, i}$ increases with $d_{i}$ increasing. From Figure 6, we can observe that the energy consumption offset increases significantly due to the increasing of $d_{i}$, while the harvested energy decreases to a low level. Furthermore, from (16), with $f_{U, i}=R_{U, i}, f_{D, i}=R_{D, i}$ and fixed time allocation, we can also calculate that $E_{O, i}$ increases with $d_{i}$ increasing, which is also in the same condition as in (1). 


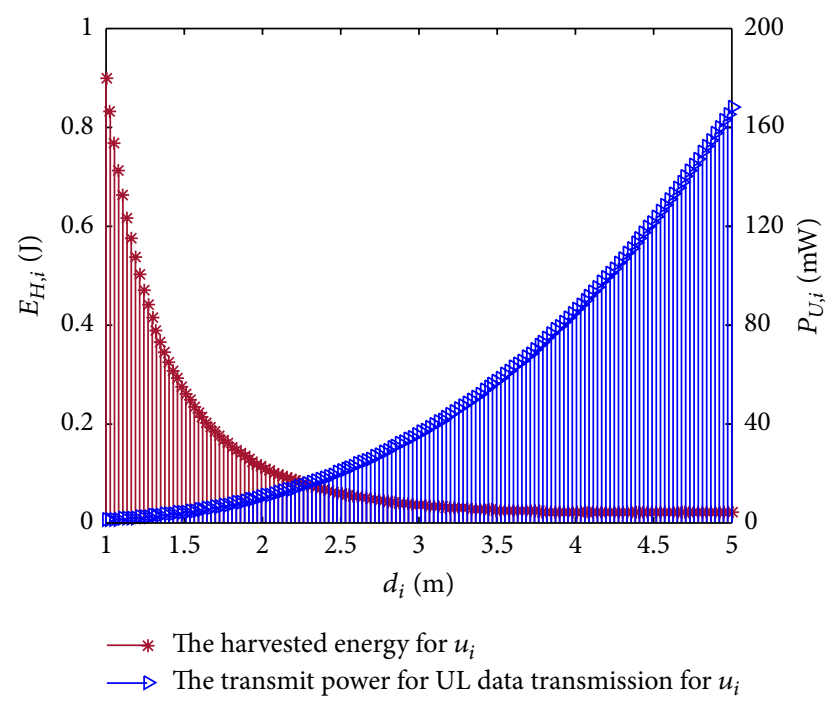

FIGURE 5: Doubly near-far problem in FD WPCN (the harvested energy for $u_{i}$ and the $u_{i}$ 's transmit power for UL data transmission, given fixed transmission rate $R_{U, i}$ ).

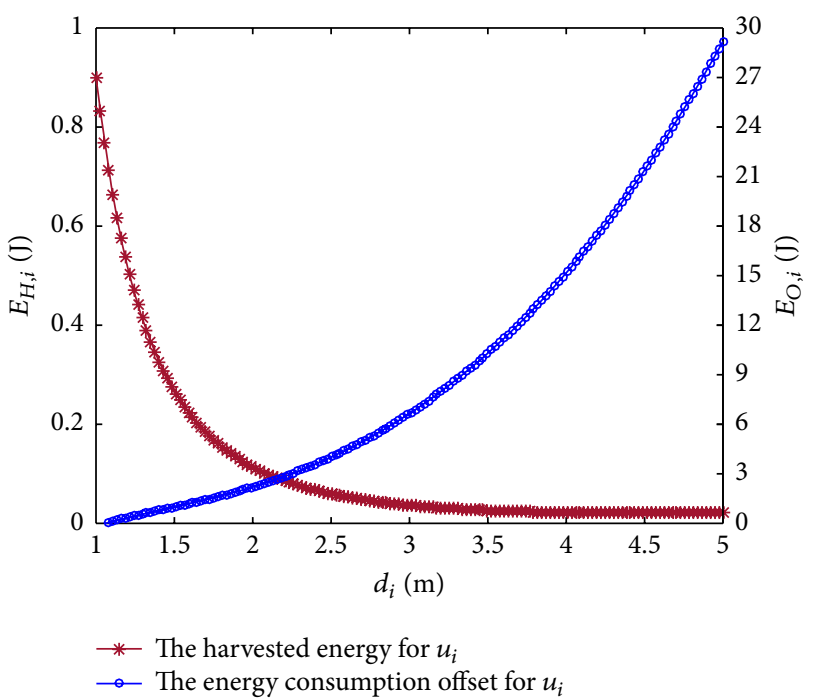

FIGURE 6: Doubly near-far problem in FD WPCN (the harvested energy for $u_{i}$ and the energy consumption offset for $u_{i}$ ).

To tackle the doubly near-far problem in FD WPCN, several effective methods have been proposed [4]. First, since TDMA protocol is adopted in the phase of UL information transmission, the far users could occupy a longer data transmission time, which can compensate the users that starved of rate. Second, space-division multiple access (SDMA) can be adopted at $\mathrm{H}$-AP with multiple antennas equipped. The near-far problem can be mitigated by controlling the transmit power of DL energy broadcasting and UL data transmission. Finally, through user cooperation, doubly near-far problem can be effectively tackled, where the resource is carefully allocated as the cooperation [4].
Fairness should be considered as an importance issue when doubly near-far problem is tackled in FD WPCN. It is well known that the same data transmission bandwidth for each user can achieve equal throughput, which is referred to as throughput-based fairness. Specifically, we have

$$
b_{i}=T_{U, i} R_{U, i} .
$$

As shown in (17), throughput-based fairness requires that users with lower rates (users far away from H-AP) occupy the medium for longer transmission time than those with higher rates, which can lead to a drastically decline in network throughput (sum of bandwidth). In [17], the authors discussed it in detail and proposed a performance metric referred to as common-throughput. Similarly, timebased fairness, which represents that each user has an equal opportunity of transmission time to occupy channel and outperforms throughput-based fairness in multirate wireless networks, is proposed to overcome this problem. Moreover, tradeoff between fairness and throughput is resolved with the introduction of proportional fairness in FD WPCN, which implements time-based fairness $[28,29]$.

4.3. Problem Definition. In this subsection, to tackle the doubly near-far problem in FD WPCN, we aim to maximize the total bandwidth utility for proportional fairness with as little energy consumption offset as possible, which is formulated as the following optimization problem:

$$
\begin{array}{rl}
(P 1): \max _{\mathbf{P}, \mathbf{T}} & F(\mathbf{P}, \mathbf{T}) \\
\min _{\mathbf{P}, \mathbf{T}} & E_{O, N}(\mathbf{P}, \mathbf{T}) \\
\text { s.t. } & 0 \leq P_{U, i} \leq P_{U, \max } \\
& T_{D, B C}+\sum_{i=1}^{M} T_{U, i}+T_{D, A}=T \\
& T_{n} \leq T_{U, i} \\
& i \in[1, M],
\end{array}
$$

where (18a) represents the objective function for proportional fairness in FD WPCN. From (7) and (17), $F(\mathbf{P}, \mathbf{T})$ can be denoted by

$$
F(\mathbf{P}, \mathbf{T})=\sum_{i=1}^{M} \omega_{i} \log \left(T_{U, i} R_{U, i}\right) .
$$

Moreover, (18b) is the objective function for minimizing the energy consumption offset for entire network, which can be energy efficient. Inequality $(18 \mathrm{c})$ is the transmit power constraint. Equality (18d) and inequality (18e) represent the time constraints.

Lemma 1. $F(\mathbf{P}, \mathbf{T})$ is concave function, and $E_{O, N}(\mathbf{P}, \mathbf{T})$ is convex function of $(\mathbf{P}, \mathbf{T})$ for $i \in[1, M]$.

Proof. Please refer to Appendix A for details. 


\section{Solution and Proposed Algorithm}

In this section, we tackle $(P 1)$ by jointly power and time allocation, which is to maximize bandwidth utility for proportional fairness while controlling the energy consumption offset to a minimum value. Since $(P 1)$ has two objective functions, we can convert it to $(P 2)$, which has one objective function. Moreover, $(P 2)$ can be proved to be convex optimization problem and can be solved by Karush-Kuhn-Tucker (KKT) conditions, with Slater's condition satisfied [33]. Based on the steps of convex optimization techniques, we can find an optimal time and transmit power allocation and propose an algorithm to describe it.

Specifically, we use the method of weighted sum of objectives as one single objective function to formulate a new objective function, which can be denoted by

$$
f(\mathbf{P}, \mathbf{T})=a_{1} F(\mathbf{P}, \mathbf{T})-a_{2} E_{O, N}(\mathbf{P}, \mathbf{T}),
$$

where $a_{1}$ and $a_{2}$ are the weight of proportional fairness utility function and energy consumption offset function. Besides, both of them can be normalized coefficients and can be set by utilizing experts experience. In addition, to ensure (20) to be a concave function, we have

$$
\begin{aligned}
& f\left[\left(\theta \mathbf{P}_{1}+(1-\theta) \mathbf{P}_{2}, \theta \mathbf{T}_{1}+(1-\theta) \mathbf{T}_{2}\right)\right] \\
& \leq \theta f\left(\mathbf{P}_{1}, \mathbf{T}_{1}\right)+(1-\theta) f\left(\mathbf{P}_{2}, \mathbf{T}_{2}\right) \Longleftrightarrow \\
& \frac{f_{1}}{f_{2}} \leq \frac{a_{2}}{a_{1}},
\end{aligned}
$$

where $\theta$ is an arbitrary real number and $\left(\mathbf{P}_{1}, \mathbf{T}_{1}\right)$ and $\left(\mathbf{P}_{2}, \mathbf{T}_{2}\right)$ are arbitrary elements in the domain of function $f$. Specifically, $f_{1}$ and $f_{2}$ are written by

$$
\begin{aligned}
f_{1}= & F\left[\left(\theta \mathbf{P}_{1}+(1-\theta) \mathbf{P}_{2}, \theta \mathbf{T}_{1}+(1-\theta) \mathbf{T}_{2}\right)\right] \\
& -\theta F\left(\mathbf{P}_{1}, \mathbf{T}_{1}\right)-(1-\theta) F\left(\mathbf{P}_{2}, \mathbf{T}_{2}\right), \\
f_{2}= & E_{O, N}\left[\left(\theta \mathbf{P}_{1}+(1-\theta) \mathbf{P}_{2}, \theta \mathbf{T}_{1}+(1-\theta) \mathbf{T}_{2}\right)\right] \\
& -\theta E_{O, N}\left(\mathbf{P}_{1}, \mathbf{T}_{1}\right)-(1-\theta) E_{O, N}\left(\mathbf{P}_{2}, \mathbf{T}_{2}\right) .
\end{aligned}
$$

If (21) is satisfied, (20) can be proved to be concave function [33]. Furthermore, we assume that $a_{1}$ and $a_{2}$ satisfied this condition when they are set. Thus, the one objective optimization problem is formulated as

$$
\begin{array}{ll}
(P 2): \max _{\mathbf{P}, \mathbf{T}} & f(\mathbf{P}, \mathbf{T}) \\
\text { s.t. } & 0 \leq P_{U, i} \leq P_{U, \max } \\
& T_{D, B C}+\sum_{i=1}^{M} T_{U, i}+T_{D, A}=T \\
& T_{n} \leq T_{U, i} \\
& i \in[1, M] .
\end{array}
$$

Proposition 2. $(P 2)$ is a convex optimization problem and can be figured out by using convex optimization techniques.
Proof. We set $a_{1}$ and $a_{2}$ according to (21). Thus, (20) is concave function. In addition, we can also observed that all the constraints of $(P 2)$ are affine. Thus, $(P 2)$ is a convex optimization problem. Also, the optimal solution of $(P 2)$ can be obtained by using convex optimization techniques.

Optimization problem $(P 2)$ can be solved with Lagrangian, which can be expressed as

$$
\begin{aligned}
\mathscr{L}(\mathbf{P}, \mathbf{T}, \boldsymbol{\lambda}, \mu)= & a_{1} F(\mathbf{P}, \mathbf{T})-a_{2} E_{O, N}(\mathbf{P}, \mathbf{T}) \\
& -\sum_{i=1}^{M} \lambda_{1 P, i}\left(P_{U, i}-P_{U, \max }\right) \\
& +\sum_{i=1}^{M} \lambda_{2 P, i} P_{U, i}+\sum_{i=1}^{M} \lambda_{T, i}\left(T_{U, i}-T_{n}\right) \\
& -\mu\left(\sum_{i=1}^{M} T_{U, i}+T_{D, B C}+T_{D, A}-T\right),
\end{aligned}
$$

where $\lambda \triangleq\left\{\lambda_{1 P, i}, \lambda_{2 P, i}, \lambda_{T, i}\right\}$ and $\mu$ denote the Lagrange multipliers associated with the right-hand side of constraint (23b), left-hand side of constraint (23b), constraint (23d), and constraint (23c), respectively. Specifically, $\lambda, \mu \geq 0$. The dual function of problem (P2) is denoted by

$$
\mathscr{G}(\lambda, \mu)=\max _{\mathbf{P}, \mathbf{T} \in \mathscr{D}} \mathscr{L}(\mathbf{P}, \mathbf{T}, \lambda, \mu),
$$

where $\mathscr{D}$ is a feasible set of $(\mathbf{P}, \mathbf{T})$ as $(23 \mathrm{~b}),(23 \mathrm{c})$, and $(23 \mathrm{~d})$ indicating. It can be observed that there exists $(\mathbf{P}, \mathbf{T})$ in $\mathscr{D}$ satisfying $0<P_{U, i}<P_{U, \max }, T_{n}<T_{U, i}$. Thus, Slater's condition is satisfied [33]. Moreover, the dual problem of (P2) can be expressed as

$$
\min _{\lambda, \mu} \mathscr{G}(\lambda, \mu)
$$

Proposition 3. The optimal solution of $(P 2)$ is given by $\mathbf{P}^{*} \triangleq$ $\left\{P_{U, i}^{*}\right\}$ and $\mathbf{T}^{*} \triangleq\left\{T_{U, i}^{*}\right\}, i \in[1, M]$, where

$$
\begin{aligned}
& P_{U, i}^{*}=\min \left[\left(\frac{\delta_{i}^{*}-1}{k_{U, i}}\right)^{+}, P_{U, \max }\right], \\
& T_{U, i}^{*}=\max \left[\left(\frac{a_{1} \omega_{i} \ln 2}{a_{2}\left(C\left(P_{U, i}\right)+\phi_{S I C, i} P_{U, i}\right)+\mu}\right)^{+}, T_{n}\right],
\end{aligned}
$$

with

$$
\begin{aligned}
k_{U, i} & =\frac{h_{U, i}}{P_{B} S_{B}+\sigma^{2}}, \\
C\left(P_{U, i}\right) & =\left(\beta_{1} P_{U, i}+\beta_{2} d_{i}^{\alpha}+\rho_{U, i}\right) \ln \left(1+k_{U, i} P_{U, i}\right),
\end{aligned}
$$

and $\delta_{i}^{*}$ is the solution of $f\left(\delta_{i}\right)=a_{2}\left(\phi_{S I C, i} T_{U, i}+T_{B} \eta_{D} S_{D}\right)$, where

$$
\begin{aligned}
f(\delta)= & \frac{a_{1} \omega_{i} \ln 2 k_{U, i}}{T_{U, i} R_{U, i}} \frac{1}{\delta \ln \delta}-a_{2} \beta_{1} T_{U, i} \ln \delta \\
& -a_{2} T_{U, i} \beta_{1} \frac{\delta-1}{\delta}-a_{2} T_{U, i}\left(\beta_{2} d_{i}^{\alpha}+\rho_{U, i}\right) \frac{k_{U, i}}{\delta} .
\end{aligned}
$$


(1) Initialize $j=1, \mu_{0}, \mu_{1}$. ( $\mu_{j}$ denotes the value of $\mu$ at $j$ th iteration).

(2) while $\left|\mu_{j}-\mu_{j-1}\right|>$ predefined accuracy do

(3) Initialize $P_{U, i}$ and $T_{U, i}, i \in[1, M]$;

(4) repeat

(5) Compute $k_{U, i}$ by (29);

(6) Compute $C\left(P_{U, i}\right)$ by (30);

(7) Find $\delta_{i}$ with (31);

(8) $\quad$ Set $\delta_{i}^{*}=\delta_{i}$.

(9) Compute $P_{U, i}$ by (27).

(10) Compute $T_{U, i}$ by (28).

(11) until $P_{U, i}$ and $T_{U, i}$ converge.

(12) $j=j+1$;

(13) Update $\mu_{j}$ with mathematical method.

(14) end while

(15) Set $P_{U, i}^{*}=P_{U, i}, T_{U, i}^{*}=T_{U, i}, i \in[1, M]$.

(16) return $P_{U, i}^{*}, T_{U, i}^{*}, i \in[1, M]$;

Algorithm 1: Joint power and time allocation algorithm.

Proof. Please refer to Appendix B for details.

Based on Proposition 3, we can obtain the optimal solution of $(P 2)$. Furthermore, we can formulate the algorithm with power and time allocation, which can be shown in Algorithm 1.

According to Algorithm 1, we solve this problem by optimization techniques. We can observe from (27) that we need $\delta_{i}^{*}$ and $k_{U, i}$ to compute $P_{U, i}^{*}$. It is observed that we need $C\left(P_{U, i}\right)$ and $P_{U, i}$ to obtain $T_{U, i}^{*}$ from (28). We can obtain $\delta_{i}^{*}, k_{U, i}$, and $C\left(P_{U, i}\right)$ from (31), (29), and (30), respectively. Moreover, $P_{U, i}$ and $T_{U, i}$ are obtained by iteration until they converge. In addition, $\mu$ is needed when computing $T_{U, i}^{*}$. $\mu$ is updated with mathematical method until a predefined accuracy is reached. In Algorithm 1, we first compute the auxiliary terms for convenience of computing, which can be shown in lines $5 \sim 8$. Then, as shown in lines $4 \sim 11$, with initialized $P_{U, i}$ and $T_{U, i}$, we compute $P_{U, i}$ and $T_{U, i}$ at one time with one of them fixed. We then obtain $P_{U, i}$ and $T_{U, i}$ iteratively by (27) and (28), respectively, until they converge. In lines 2 and $12 \sim 14, \mu$ is adjusting at each iteration with subgradient algorithms of $\mathscr{G}(\lambda, \mu)$ [17] until it converges. One effective way is to utilize ellipsoid method $[17,33]$. Finally, we can obtain the optimal solution of power and time allocation for each $i \in[1, M]$.

This algorithm is finite and can have an optimal result. Since (20) is concave, $P_{U, i}, T_{U, i}, \mu$ will converge to the optimal solution of $(P 2)$. For this algorithm, the loops of steps $4 \sim 11$ and $2 \sim 14$ can have an end. The algorithm has an optimal $P_{U, i}^{*}, T_{U, i}^{*}$ return for each $i \in[1, M]$.

The computational time of steps $3 \sim 11$ is $\mathcal{O}(M)$, while those of step 13 is $\mathcal{O}(1)$, with $\mu_{j}$ being updated by the ellipsoid algorithm [17]. Overall, the time complexity of Algorithm 1 is $\mathscr{O}(M)$. Because the computational time of conversation from $(P 1)$ to $(P 2)$ is $\mathcal{O}(1)$, the time complexity for solving optimization problem $(P 1)$ is still $\mathscr{O}(M)$. Specifically, the optimization problem can be figured out in finite time with Matlab Optimization Toolbox.

\section{Simulation Results}

In this section, we provide numerical results to evaluate the performance of proposed algorithm. First, in FD WPCN, we compare the proposed algorithm, sum-throughput in $[7,17]$, and common-throughput in [17], with different number of users. Furthermore, we also compare those with different $P_{B}$. It should be pointed out that the three different strategies to tackle with doubly near-far problem are compared with the same constraints in $(P 2)$.

We set some of the parameters as follows: $T=1[\mathrm{~s}]$, $T_{D, B C}=0.05[\mathrm{~s}]$, and $T_{D, A}=0.05[\mathrm{~s}]$; the maximum transmit power $P_{U, \max }=35[\mathrm{~mW}]$; the channel power gains $g_{i}, h_{U, i}$, and $h_{D, i}$ are modeled as Rayleigh fading, with the path loss exponent $\alpha=3$. The distance $d_{i}$ is assumed to be randomly distributed between $s$ and $u_{i}$, with $1 \leq$ $d_{i} \leq 5[\mathrm{~m}]$. The different distance from H-AP to users can be shown in Figure 7. The users far away from H-AP get low throughput, low harvested energy, and high energy consumption for information transmission, compared with those located near to H-AP. With random variable, the simulation is evaluated over 1000 times average. We assume that the energy harvesting efficiency $\eta_{i}$ is the same for all users and is set to 0.5 . Also, the noise power is assumed to be $\sigma^{2}=$ $-80[\mathrm{dBm}]$. To make a comparison, we select Jain's index as fairness measurement, throughput for each user, and energy consumption offset for network as performance metrics.

In Figure 8, we investigate the effect of different number of users on fairness, with Jain's index comparison for three difference strategies: the proposed algorithm, sumthroughput, and common-throughput. It is observed that the proposed algorithm has more fluent Jain's index than common-throughput. Jain's index of common-throughput 


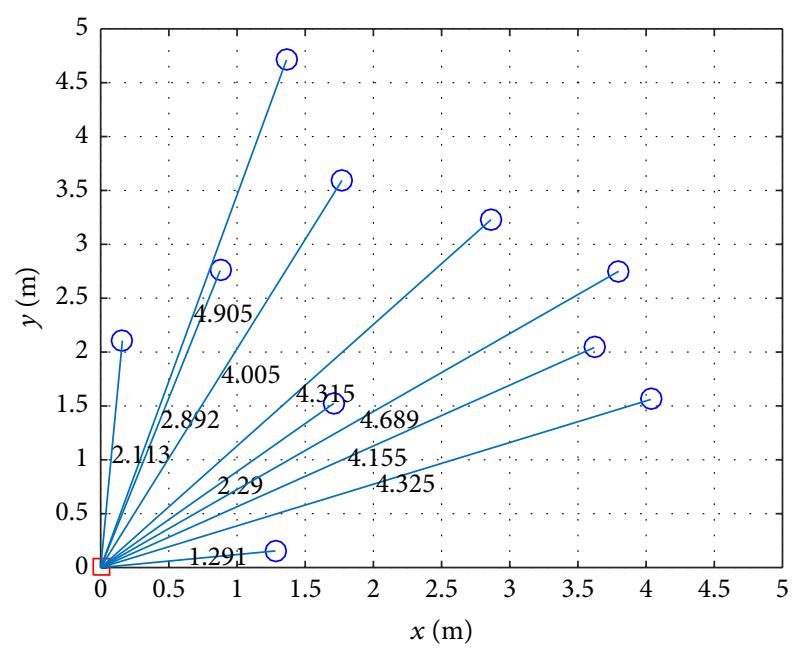

\section{$\square \mathrm{H}-\mathrm{AP}$ \\ User}

Figure 7: One H-AP and 10 users with different distance from H-AP.

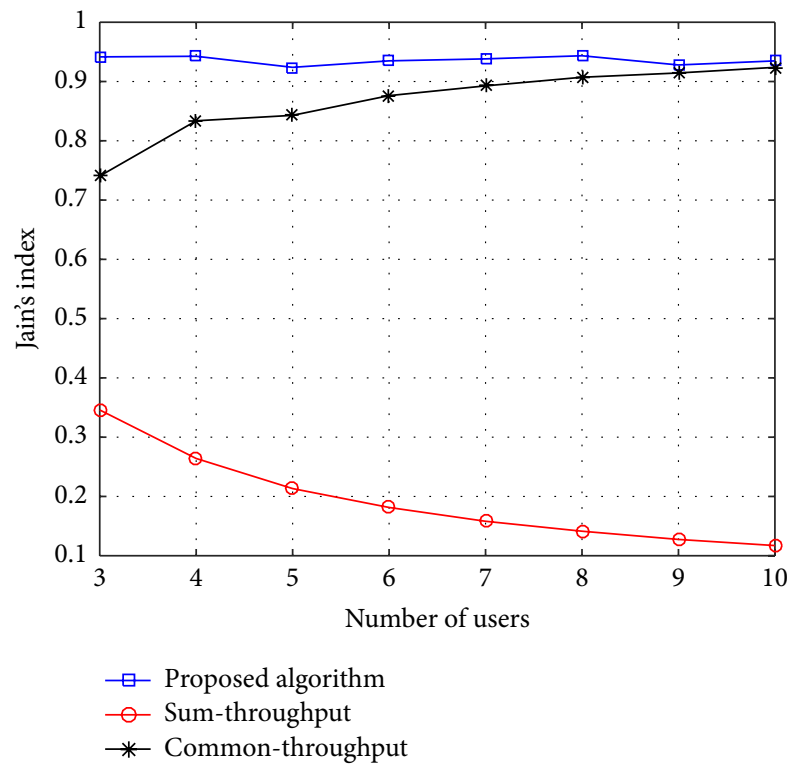

FIGURE 8: Jain's index with different number of users.

increases with the number of users increasing, while sumthroughput is decreasing. It is observed that the FD WPCN in sum-throughput situation suffers from a severe unfair resource assignment. In addition, we can observed that the proposed algorithm has the highest value of Jain's index among the three compared strategies, which represents that the proposed algorithm achieves a more fair allocation than the other two compared strategies. This indicates that the proposed algorithm performs better than common-throughput and sum-throughput in terms of Jain's index.

From Figure 9, we can observe that the proposed algorithm achieves the lowest throughput among the three strategies, with the number of users increasing. This is due to the fact that the objective function of the proposed

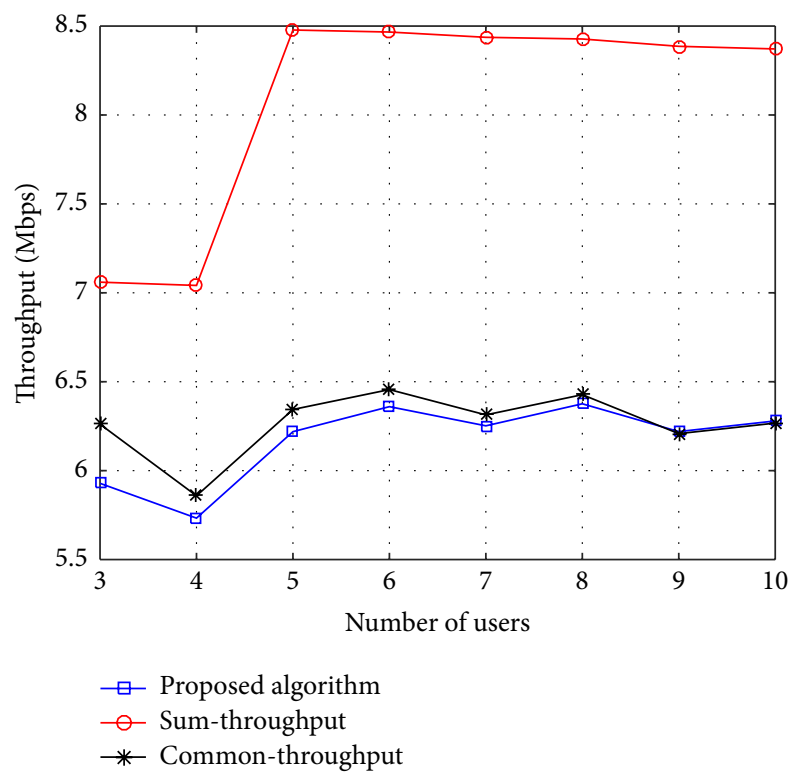

FIgURE 9: Throughput with different number of users.

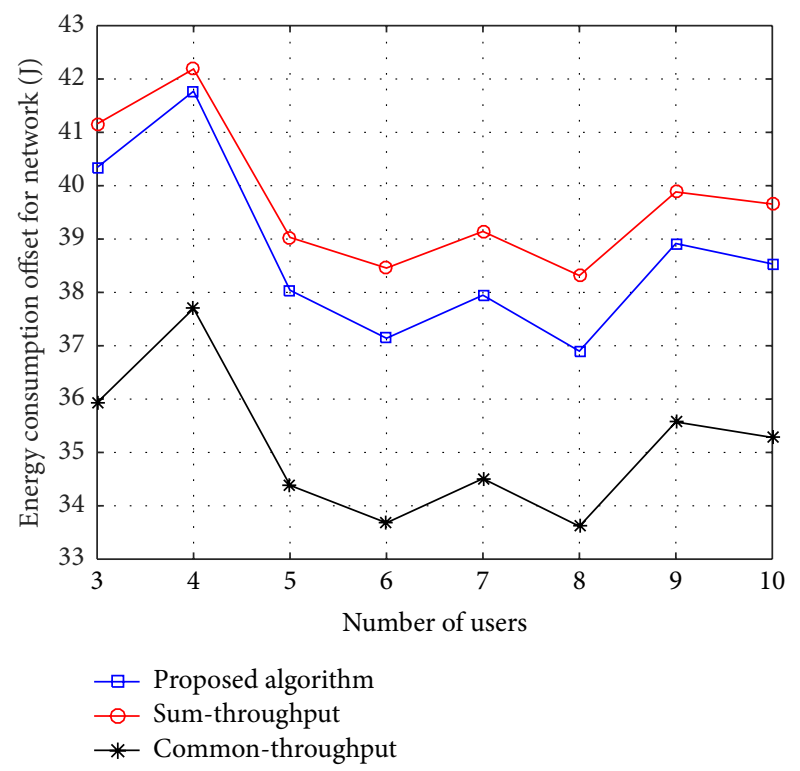

FIGURE 10: Energy consumption offset for network with different number of users.

algorithm is to achieve proportional fairness, which will find a tradeoff between fairness and throughput. While aiming at finding a fairness allocation to tackle doubly near-far problem, it performs worse over throughput than those of sum-throughput and common-throughput. Moreover, it is observed that there are some fluctuations among the three different strategies. It indicates that the number of users has a great impact on the throughput, based on the fact that different numbers of users have different time slot allocation, which can bring a throughput fluctuation.

Meanwhile, in Figure 10, we compare the three strategies with energy consumption offset for network. It is observed 


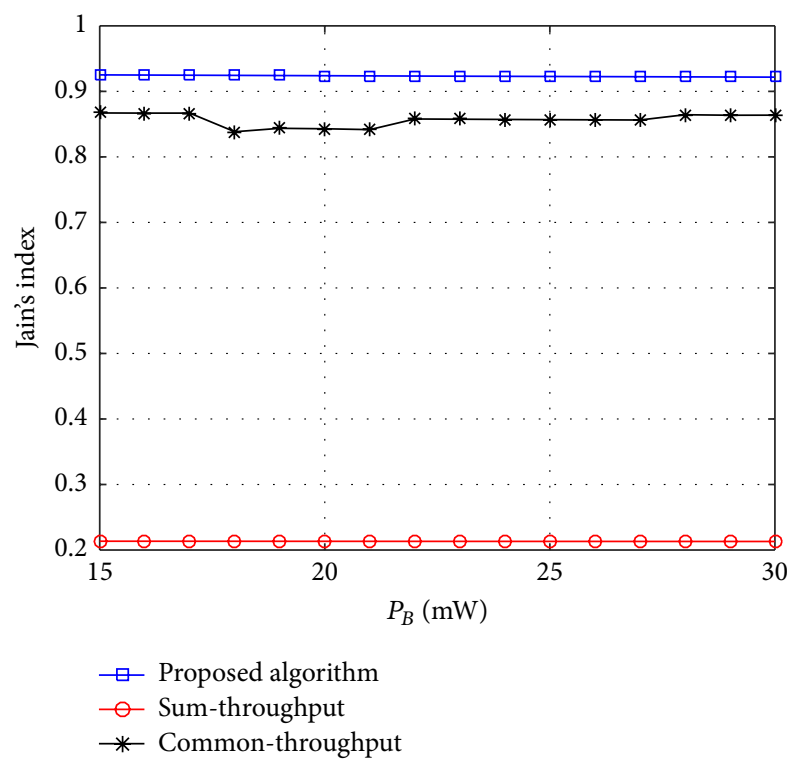

FIGURE 11: Jain's index with different transmit power of energy broadcasting.

that the value of proposed algorithm is smaller than those of sum-throughput, but greater than those of commonthroughput. It indicates that the proposed algorithm performs better than sum-throughput, but worse than commonthroughput. The reason is that minimizing energy consumption is a secondary objective function for $(P 2)$. Moreover, it can also observed that there are some fluctuations with different number of users. The fact lies in that different number of users have different allocation of transmit power, which has a great impact on the energy consumption. From Figures 8,9 , and 10 , with different number of users, we can conclude that the proposed algorithm performs better than the other two strategies on Jain's fairness index, while controlling the energy consumption offset for network to a low level.

In Figure 11, we evaluate the performance of the three different strategies on Jain's index with different transmit power of energy broadcasting. It is observed that Jain's index of three strategies are fluent with $P_{B}$ increasing. Also, the proposed algorithm has the highest value of Jain's index among the three strategies. The reason is that the transmit power of energy broadcasting has little effect on Jain's index. Jain's index is mainly related to the transmit power and time allocation for users. From Figure 11, it can be concluded that the proposed algorithm performs better than sumthroughput and common-throughput on Jain's index, with different transmit power of energy broadcasting.

Figure 12 shows the throughput comparison for different transmit power of energy broadcasting. The throughput of three strategies decreases linearly with increasing transmit power of energy broadcasting. This is for the reason that the increasing of transmit power results in an increasing interference and, furthermore, causes a decreasing UL data transmission rate. But the performance of proposed algorithm in terms of throughput is worse than sum-throughput

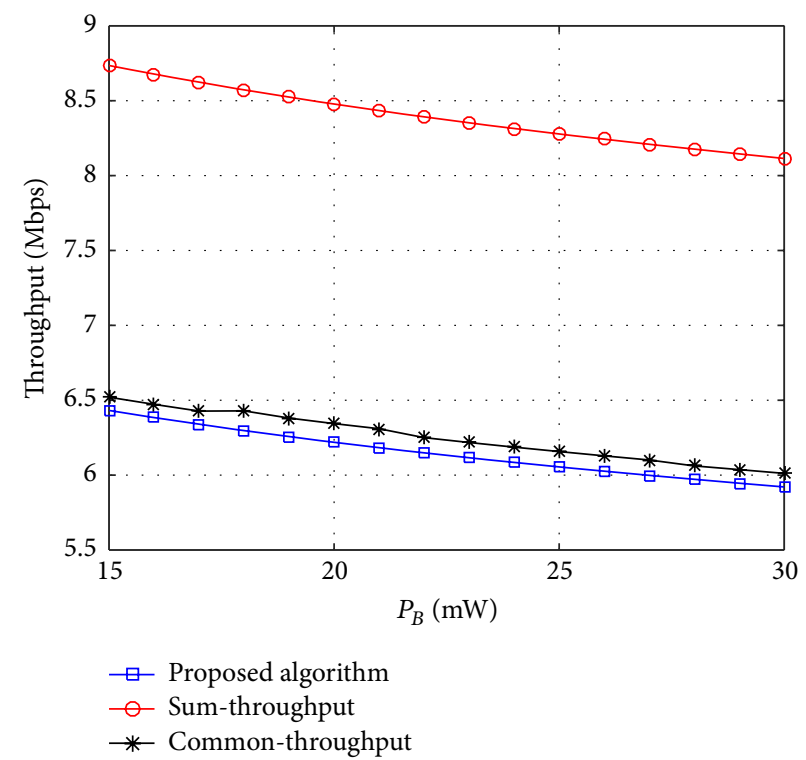

FIGURE 12: Throughput with different transmit power of energy broadcasting.

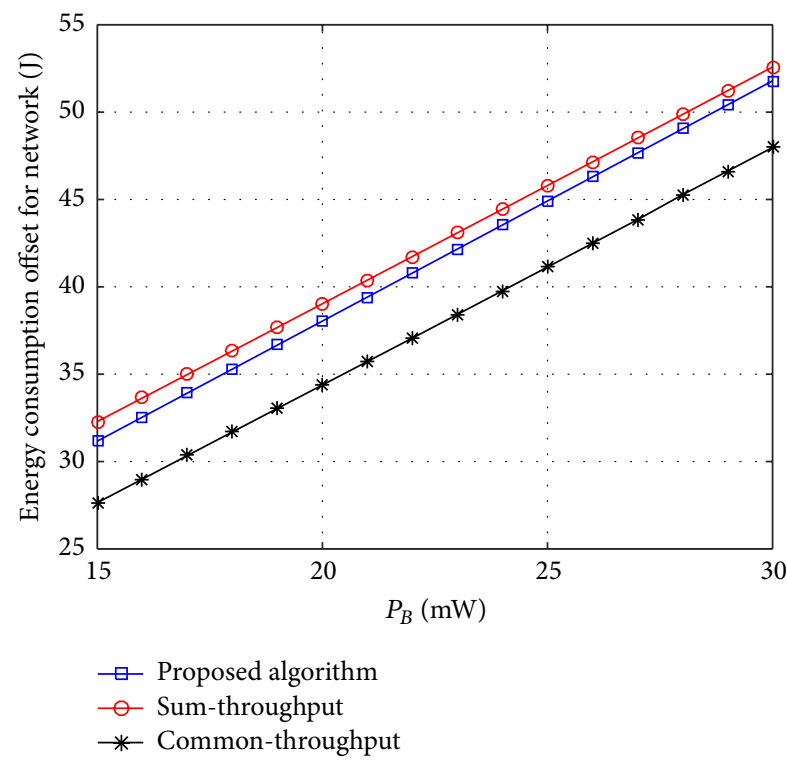

FIGURE 13: Energy consumption offset for network with different transmit power of energy broadcasting.

and common-throughput. This is also for the reason that proportional fairness brings a tradeoff between fairness and throughput and fairness turns into a key factor for the proposed algorithm.

From Figure 13, the energy consumption offset for network increases linearly with transmit power of energy broadcasting increasing. The larger the transmit power of energy broadcasting, the more the energy consumption offset for network. In addition, it is observed that the energy consumption offset for network of proposed algorithm is between those of sum-throughput and common-throughput, 
because of the fact that minimizing energy consumption offset for network turns into a secondary factor, when $a_{1}$ and $a_{2}$ are set. It indicates that the proposed algorithm outperforms the sum-throughput but performs worse than common-throughput. Similarly, from Figures 11, 12, and 13, with different transmit power of energy broadcasting, we can conclude that the proposed algorithm finds a proper fairness resource allocation, while minimizing the energy consumption offset for network to a intermediate level.

\section{Conclusion}

In this paper, we investigated the problem of optimal transmit power and time allocation for each user in the FD WPCN, which aims at achieving proportional fairness and energy efficiency. With the proposed time-division based protocol, an optimization problem was formulated to tackle the problem of unfairness resource allocation among users, while minimizing the energy consumption offset for network. With convex techniques, an optimal algorithm was proposed to solve the optimization problem, which had been proved to be convex problem. Simulation results demonstrated that the proposed algorithm outperforms other compared strategies in terms of fairness metric and plays a positive role in reducing energy consumption offset for network.

\section{Appendix}

\section{A. Proof of Lemma 1}

First, we need to prove that $F(\mathbf{P}, \mathbf{T})$ is a concave function. For $\sum \omega_{i} \log \left(T_{U, i} R_{U, i}\right)$, it is a concave function if $T_{U, i} R_{U, i}$ is a convex function, since it is the weighted logarithm summation of $T_{U, i} R_{U, i}$, while logarithm is concave and $T_{U, i} R_{U, i}>0$ [33]. It can be observed from (1) that $R_{U, i}>0$ since $h_{U, i} P_{U, i} /\left(P_{B} S_{B}+\right.$ $\left.\sigma^{2}\right)>0$.

Denote the Hessian of $T_{U, i} R_{U, i}$ by $\mathbf{H}_{i} \triangleq \nabla^{2}\left(T_{U, i} P_{U, i}\right)$ for $(\mathbf{P}, \mathbf{T})$, whose element can be expressed as $a_{m, n}^{(i)}$ at the $m$ th row and $n$th column [7, 17], $1 \leq m, n \leq 2 M$. The diagonal entries of $\mathbf{H}_{i}$ can be expressed as

$$
a_{m, m}^{(i)}= \begin{cases}\frac{-T_{U, i} k_{i}^{2}}{\left(1+k_{i} P_{U, i}\right)^{2}}, & m=i \\ 0, & \text { otherwise }\end{cases}
$$

where $k_{i}=h_{U, i} /\left(P_{B} S_{B}+\sigma^{2}\right)$. Moreover, the off-diagonal entries of $\mathbf{H}_{i}$ can be denoted by

$$
a_{m, n}^{(i)}=a_{n, m}^{(i)}= \begin{cases}\frac{k_{i}}{\left(1+k_{i} P_{U, i}\right)}, & m-M=i, n=1 \\ 0, & \text { otherwise. }\end{cases}
$$

For an arbitrary real vector

$$
\mathbf{v}=\left[P_{U, 1}, P_{U, 2}, \ldots, P_{U, M}, T_{U, 1}, T_{U, 2}, \ldots, T_{U, M}\right]^{T}
$$

since $P_{U, i}, T_{U, i}>0$, it follows from (A.1) and (A.2) that

$$
\begin{aligned}
\mathbf{v}^{T} \nabla^{2}\left(T_{U, i} P_{U, i}\right) \mathbf{v}=\mathbf{v}^{T} \mathbf{H}_{i} \mathbf{v} & \\
= & \left(\frac{-T_{U, i} k_{i}^{2} P_{U, i}}{\left(1+k_{i} P_{U, i}\right)^{2}}+\frac{k_{i} T_{U, i}}{1+k_{i} P_{U, i}}\right) P_{U, i} \\
& +\frac{k_{i} P_{U, i}}{1+k_{i} P_{U, i}} T_{U, i}=\frac{-T_{U, i} k_{i}^{2} P_{U, i}^{2}}{\left(1+k_{i} P_{U, i}\right)^{2}}+\frac{2 k_{i} P_{U, i} T_{U, i}}{1+k_{i} P_{U, i}} \\
= & \frac{2 k_{i} P_{U, i} T_{U, i}+T_{U, i} k_{i}^{2} P_{U, i}^{2}}{\left(1+k_{i} P_{U, i}\right)^{2}} \geq 0 .
\end{aligned}
$$

Thus, $\mathbf{H}_{i}$ is a semidefinite matrix. Therefore, $T_{U, i} P_{U, i}$ is a convex function [33]. Furthermore, this completes the proof of $F(\mathbf{P}, \mathbf{T})$ which is a concave function.

Second, we need to prove that $E_{O, N}(\mathbf{P}, \mathbf{T})$ is a convex function for $(\mathbf{P}, \mathbf{T})$; we can prove it by

$$
\begin{aligned}
& E_{O, N}\left[\theta\left(\mathbf{P}_{1}, \mathbf{T}_{1}\right)+(1-\theta)\left(\mathbf{P}_{2}, \mathbf{T}_{2}\right)\right] \\
& \quad \leq \theta E_{O, N}\left(\mathbf{P}_{1}, \mathbf{T}_{1}\right)+(1-\theta) E_{O, N}\left(\mathbf{P}_{2}, \mathbf{T}_{2}\right),
\end{aligned}
$$

where $\theta$ is an arbitrary number with $0 \leq \theta \leq 1$ and the $\operatorname{arbitrary}\left(\mathbf{P}_{1}, \mathbf{T}_{1}\right),\left(\mathbf{P}_{2}, \mathbf{T}_{2}\right) \in \operatorname{dom}\left(E_{O, N}\right)$. If (A.5) is satisfied, $E_{O, N}(\mathbf{P}, \mathbf{T})$ can be proved to be a convex function [33]. It can be followed that

$$
\begin{aligned}
& E_{\mathrm{O}, N} {\left[\left(\theta \mathbf{P}_{1}+(1-\theta) \mathbf{P}_{2}, \theta \mathbf{T}_{1}+(1-\theta) \mathbf{T}_{2}\right)\right] } \\
&- \theta E_{\mathrm{O}, N}\left(\mathbf{P}_{1}, \mathbf{T}_{1}\right)-(1-\theta) E_{\mathrm{O}, N}\left(\mathbf{P}_{2}, \mathbf{T}_{2}\right) \\
&= \sum_{i=1}^{M} \phi_{\mathrm{SIC}, i} \\
& \cdot\left[\theta P_{U 1, i}(1-\theta) T_{U 2, i}+(1-\theta) P_{U 2, i} \theta T_{U 1, i}\right] \\
&-\sum_{i=1}^{M}\left(\left(\sum_{i=1}^{M} \theta T_{U 1, i}\right)(1-\theta) \eta_{D} S_{D} P_{U 2, i}\right) \\
&-\sum_{i=1}^{M}\left(\left(\sum_{i=1}^{M}(1-\theta) T_{U 1, i}\right) \eta_{i} g_{i} P_{B} \theta P_{U 1, i}\right)<0 ;
\end{aligned}
$$

therefore, $E_{O, N}(\mathbf{P}, \mathbf{T})$ is a convex function for $(\mathbf{P}, \mathbf{T})$.

Finally, we have proved that $F(\mathbf{P}, \mathbf{T})$ and $E_{O, N}(\mathbf{P}, \mathbf{T})$ are convex of $(\mathbf{P}, \mathbf{T})$ for $i \in[1, M]$. This completes the proof of Lemma 1.

\section{B. Proof of Proposition 3}

Since $\mathbf{T}$ do not appear in (23b) in (P2). (P2) can be decomposed into two optimization problems: the first one 
optimizes $\mathbf{T}$ and the second one optimizes $\mathbf{P}$ [34]. The first optimization problems can be expressed as

$$
\begin{array}{ll}
(P 3): \max _{\mathrm{T}} & (20) \\
\text { s.t. } & T_{D, B C}+\sum_{i=1}^{M} T_{U, i}+T_{D, A}=T \\
& T_{n} \leq T_{U, i}
\end{array}
$$

and the second optimization problem can be denoted by

$$
\begin{aligned}
(P 4): \max _{\mathbf{P}} & (20) \\
\text { s.t. } & 0 \leq P_{U, i} \leq P_{U, \max } .
\end{aligned}
$$

The Lagrangian of $(P 3)$ is expressed as

$$
\begin{gathered}
\mathscr{L}\left(\mathbf{T}, \lambda_{T, i}, \mu\right)=a_{1} F(\mathbf{P}, \mathbf{T})-a_{2} E_{O, N}(\mathbf{P}, \mathbf{T}) \\
+\sum_{i=1}^{M} \lambda_{T, i}\left(T_{U, i}-T_{n}\right) \\
-\mu\left(\sum_{i=1}^{M} T_{U, i}+T_{D, B C}+T_{D, A}-T\right) .
\end{gathered}
$$

From (B.1c), we can observed that there exists $\mathbf{T}$ satisfying $T_{n}<T_{U, i}$. Strong duality holds for $(P 3)$ with Slater's condition satisfied. Thus, $(P 3)$ can be figured out by using KarushKuhn-Tucker (KKT) conditions, which can be shown as

$$
\begin{aligned}
\lambda_{T, i}, \mu & \geq 0, \\
T_{n} & \leq T_{U, i}^{*}, \\
\sum_{i=1}^{M} T_{U, i}^{*}+T_{D, B C}+T_{D, A}-T & =0, \\
\lambda_{T, i}\left(T_{U, i}^{*}-T_{n}\right) & =0, \\
\left.\frac{\partial \mathscr{L}\left(T, \lambda_{T, i}, \mu\right)}{\partial T_{U, i}}\right|_{T_{U, i}=T_{U, i}^{*}} & \\
\forall i & \in[1, M],
\end{aligned}
$$

where $T_{U, i}^{*}$ are the optimal solutions of (P3). With (B.6), from (B.8), we can obtain

$$
\begin{aligned}
& \frac{a_{1} \omega_{i} \ln 2}{T_{U, i}^{*}} \\
& \quad-a_{2}\left[\left(\beta_{1} P_{U, i}+\beta_{2} d_{i}^{\alpha}+\rho_{U, i}\right) R_{U, i}-\phi_{\mathrm{SIC}, i} P_{U, i}\right] \\
& \quad+\lambda_{T, i}=\mu .
\end{aligned}
$$

Introducing $\lambda_{T, i}$ in (B.10) into (B.7), we obtain

$$
\begin{gathered}
T_{U, i}^{*}=\max \left[\left(\frac{a_{1} \omega_{i} \ln 2}{a_{2}\left(C\left(P_{U, i}\right)+\phi_{\mathrm{SIC}, i} P_{U, i}\right)+\mu}\right)^{+}, T_{n}\right], \\
\mathrm{C}\left(P_{U, i}\right)=\left(\beta_{1} P_{U, i}+\beta_{2} d_{i}^{\alpha}+\rho_{U, i}\right) \ln \left(1+\frac{h_{U, i} P_{U, i}}{P_{B} S_{B}+\sigma^{2}}\right) .
\end{gathered}
$$

With given optimized $\mathbf{T}, i \in[1, M]$. The Lagrangian of $(P 4)$ is written as

$$
\begin{aligned}
\mathscr{L}\left(\mathbf{P}, \lambda_{1 P, i}, \lambda_{2 P, i}\right)= & a_{1} F(\mathbf{P}, \mathbf{T})-a_{2} E_{O, N}(\mathbf{P}, \mathbf{T}) \\
& -\sum_{i=1}^{M} \lambda_{1 P, i}\left(P_{U, i}-P_{U, \max }\right) \\
& +\sum_{i=1}^{M} \lambda_{2 P, i} P_{U, i} .
\end{aligned}
$$

It can be observed that there exists $\mathbf{P}$ satisfying $0<P_{U, i}<$ $P_{U, \max }$. Thus, Slater's condition is satisfied [33]. (P4) can be solved by its Karush-Kuhn-Tucker (KKT) conditions [13, 17], which can be expressed as

$$
\begin{aligned}
\lambda_{1 P, i}, \lambda_{2 P, i} & \geq 0, \\
0 & \leq P_{U, i}^{*} \leq P_{U, \max }, \\
\lambda_{1 P, i}\left(P_{U, i}^{*}-P_{U, \max }\right) & =0, \\
\lambda_{2 P, i} P_{U, i}^{*} & =0, \\
\left.\frac{\partial \mathscr{L}\left(\mathbf{P}, \lambda_{1 P, i}, \lambda_{2 P, i}\right)}{\partial P_{U, i}}\right|_{P_{U, i}=P_{U, i}^{*}} & =0, \\
\forall i & \in[1, M],
\end{aligned}
$$

where $P_{U, i}^{*}, \lambda_{1 P, i}, \lambda_{2 P, i}$ denote the optimal solutions of (P4). Therefor, from (B.17), we can get

$$
\begin{aligned}
& \frac{a_{1} \omega_{i} \ln 2 R_{U, i}^{\prime}}{T_{U, i} R_{U, i}}-a_{2}\left(\phi_{\mathrm{SIC}, i} T_{U, i}+T_{B} \eta_{D} S_{D}\right) \\
& \quad-a_{2} \beta_{1} T_{U, i} R_{U, i}-a_{2} T_{U, i} \beta_{1} P_{U, i} R_{U, i}^{\prime} \\
& \quad-a_{2} T_{U, i} R_{U, i}^{\prime}\left(\beta_{2} d_{i}^{\alpha}+\rho_{U, i}\right)=\lambda_{1 P, i}-\lambda_{2 P, i} .
\end{aligned}
$$

With (B.15) and (B.16), we can obtain

$$
\begin{gathered}
P_{U, i}^{*}=\min \left[\left(\frac{\delta_{i}^{*}-1}{k_{U, i}}\right)^{+}, P_{U, \max }\right] \\
k_{U, i}=\frac{h_{U, i}}{P_{B} S_{B}+\sigma^{2}},
\end{gathered}
$$

where symbol (value $)^{+}$denotes $\max (0$, value). Specifically, we have $\delta_{i}=1+k_{U, i} P_{U, i}$. $\delta_{i}^{*}$ is the solution of

$$
f\left(\delta_{i}\right)=a_{2}\left(\phi_{\mathrm{SIC}, i} T_{U, i}+T_{B} \eta_{D} S_{D}\right),
$$

where

$$
\begin{aligned}
f(\delta)= & \frac{a_{1} \omega_{i} \ln 2 k_{U, i}}{T_{U, i} R_{U, i}} \frac{1}{\delta \ln \delta}-a_{2} \beta_{1} T_{U, i} \ln \delta \\
& -a_{2} T_{U, i} \beta_{1} \frac{\delta-1}{\delta} \\
& -a_{2} T_{U, i}\left(\beta_{2} d_{i}^{\alpha}+\rho_{U, i}\right) \frac{k_{U, i}}{\delta} .
\end{aligned}
$$

This completes the proof of Proposition 3. 


\section{Notation}

$s, u_{i}: \quad$ Hybrid access point (H-AP), user $i$

$M, U: \quad$ The number of all users, the set of all users

$d_{i}, \alpha: \quad$ The distance between $s$ and $u_{i}$, path loss exponent

$J, b_{i}: \quad$ Jain's fairness index [31], UL bandwidth for

$P_{B}$ : $\quad$ The transmit power of energy

broadcasting in DL at $s$

$P_{U, i}: \quad$ The transmit power for data transmission in UL at $u_{i}$

$P_{U, \max }: \quad$ The maximum transmit power of $P_{U, i}$

$P_{U, s}: \quad$ The received power for data transmission in UL at $s$

$P_{D, s}: \quad$ The DL transmit power for data transmission at $s$

$P_{D, i}: \quad$ The DL received power for data transmission at $u_{i}$

$g_{i}$ : The DL channel power gain for energy broadcasting from $s$ to $u_{i}$

$h_{U, i}: \quad$ The UL channel power gain for data transmission from $u_{i}$ to $s$

$h_{D, i}: \quad$ The channel power gain in DL for data transmission

$S_{B}$ : The equivalent SIC power gain at $s$ for energy broadcasting

$S_{D}: \quad$ The equivalent power gain after SIC at $u_{i}$

$\eta_{i}, \eta_{D}$ : Energy harvesting efficiency at $u_{i}$, power transfer efficiency for SIC at $u_{i}$

$R_{U, i}, R_{D, i}$ : The instantaneous data transmission rate in UL, in DL

$f_{U, i}, f_{D, i}$ : The flow rate from $u_{i}$ to $s$ in UL, from $s$ to $u_{i}$ in DL

$P_{H, i}: \quad$ The amount of power harvested at $u_{i}$

$p_{D, i}, p_{U, i}:$ The rate of energy consumption for data flow from $u_{i}$ to $s$ in UL, from $s$ to $u_{i}$ in DL

$C_{U, i}, C_{D, i}:$ The rate of energy consumption for transmitting one unit data flow in UL from $u_{i}$ to $s$, in UL from $s$ to $u_{i}$

$\rho_{U, i}, \rho_{D, i}:$ The rate of reception energy consumption for one data flow in UL from $u_{i}$ to $s$, in DL from $s$ to $u_{i}$

$\beta_{1}, \beta_{2}$ : Transmitter-related constant term, amplifier-related coefficient term

$T_{D, B C}$ : The transmission time for beacon frame and control frame

$T_{B}$ : The duration of FD phase

$T_{U, i}, T_{n}: \quad u_{i}$ 's data transmission time, least required time duration

$T_{D, A}$ : The duration for data acknowledgment transmission

T: $\quad$ The period of FD time-division based MAC protocol

$E_{c}, E_{c, i}$ : Energy consumption of network, energy consumption of $u_{i}$

$E_{\text {cal }}$ : Total energy consumption for calculation at $s$ and $u_{i}$
$E_{\mathrm{cal}_{s} s}: \quad$ Energy consumption for calculation at $s$

$E_{\text {cal }, i}: \quad$ Energy consumption for calculation at $u_{i}$

$E_{D}, E_{D, i}$ : Total energy consumption for data transmission in DL from $s$ to $u_{i}$ in total, at $u_{i}$

$E_{U}, E_{U, i}: \quad$ Energy consumption for data transmission in UL from $u_{i}$ to $s$ in total, at $u_{i}$

$E_{B}$ : $\quad$ Energy consumption for energy broadcasting at $s$

$E_{\text {SIC }} \quad$ Energy consumption for SIC in $s$ and $u_{i}$

$\phi_{\text {SIC, }}$ : Energy consumption coefficient term for SIC at $s$

$\phi_{\mathrm{SIC}, i}: \quad$ Energy consumption coefficient term for SIC at $u_{i}$

$E_{H}, E_{H, i}$ : The total harvested energy for network, for $u_{i}$

$E_{O, N}, E_{O, i}$ : The energy consumption offset for network, for $u_{i}$.

\section{Competing Interests}

The authors declare that there are no competing interests regarding the publication of this paper.

\section{References}

[1] Z. Popovic, E. A. Falkenstein, D. Costinett, and R. Zane, "Low-power far-field wireless powering for wireless sensors," Proceedings of the IEEE, vol. 101, no. 6, pp. 1397-1409, 2013.

[2] H. J. Visser and R. J. M. Vullers, "RF energy harvesting and transport for wireless sensor network applications: principles and requirements," Proceedings of the IEEE, vol. 101, no. 6, pp. 1410-1423, 2013.

[3] H. Tabassum, E. Hossain, A. Ogundipe, and D. I. Kim, "Wireless-powered cellular networks: key challenges and solution techniques," IEEE Communications Magazine, vol. 53, no. 6, pp. 63-71, 2015.

[4] S. Bi, C. K. Ho, and R. Zhang, "Wireless powered communication: opportunities and challenges," IEEE Communications Magazine, vol. 53, no. 4, pp. 117-125, 2015.

[5] J. Lorincz and I. Bule, "Renewable energy sources for power supply of base station sites," International Journal of Business Data Communications and Networking, vol. 9, no. 3, pp. 53-74, 2013.

[6] J. Lorincz, I. Bule, and M. Kapov, "Performance analyses of renewable and fuel power supply systems for different base station sites," Energies, vol. 7, no. 12, pp. 7816-7846, 2014.

[7] X. Kang, C. K. Ho, and S. Sun, "Full-duplex wireless-powered communication network with energy causality," IEEE Transactions on Wireless Communications, vol. 14, no. 10, pp. 5539-5551, 2015.

[8] B. Suzhi, H. Chin Keong, and Z. Rui, "Recent advances in joint wireless energy and information transfer," in Proceedings of the IEEE Information Theory Workshop (ITW '14), pp. 341345, Hobart, Australia, November 2014.

[9] M. Jain, J. I. Choi, T. Kim et al., "Practical, real-time, full duplex wireless," in Proceedings of the 17th Annual International Conference on Mobile Computing and Networking (MobiCom '11), pp. 301-312, ACM, Las Vegas, Nev, USA, September 2011. 
[10] A. Sabharwal, P. Schniter, D. Guo, D. W. Bliss, S. Rangarajan, and R. Wichman, "In-band full-duplex wireless: challenges and opportunities," IEEE Journal on Selected Areas in Communications, vol. 32, no. 9, pp. 1637-1652, 2014.

[11] D. Kim, H. Lee, and D. Hong, "A survey of in-band full-duplex transmission: from the perspective of PHY and MAC layers," IEEE Communications Surveys \& Tutorials, vol. 17, no. 4, pp. 2017-2046, 2015

[12] S. Leng, D. W. K. Ng, N. Zlatanov, and R. Schober, "Multiobjective resource allocation in full-duplex SWIPT systems," http://arxiv.org/abs/1510.01434.

[13] H. Ju and R. Zhang, "Optimal resource allocation in full-duplex wireless-powered communication network," IEEE Transactions on Communications, vol. 62, no. 10, pp. 3528-3540, 2014.

[14] H. Ju, K. Chang, and M.-S. Lee, "In-band full-duplex wireless powered communication networks," in Proceedings of the 17th IEEE International Conference on Advanced Communications Technology (ICACT '15), pp. 23-27, Seoul, Republic of Korea, July 2015.

[15] M. Gao, H. H. Chen, Y. Li, M. Shirvanimoghaddam, and J. Shi, "Full-duplex wireless-powered communication with antenna pair selection," in Proceedings of the IEEE Wireless Communications and Networking Conference (WCNC '15), pp. 693-698, IEEE, New Orleans, La, USA, March 2015.

[16] Y. Zeng and R. Zhang, "Full-duplex wireless-powered relay with self-energy recycling," IEEE Wireless Communications Letters, vol. 4, no. 2, pp. 201-204, 2015.

[17] H. Ju and R. Zhang, "Throughput maximization in wireless powered communication networks," IEEE Transactions on Wireless Communications, vol. 13, no. 1, pp. 418-428, 2014.

[18] S. Bi, Y. Zeng, and R. Zhang, "Wireless powered communication networks: an overview," IEEE Wireless Communications, vol. 23, no. 2, pp. 10-18, 2016.

[19] H. Chingoska, I. Nikoloska, Z. Hadzi-Velkov, and N. Zlatanov, "Proportional fair scheduling in wireless powered communication networks," in Proceedings of the 23rd International Conference on Telecommunications (ICT '16), pp. 1-5, Thessaloniki, Greece, May 2016.

[20] C. Guo, B. Liao, L. Huang, Q. Li, and X. Lin, "Convexity of fairnessaware resource allocation in wireless powered communication networks," IEEE Communications Letters, vol. 20, no. 3 , pp. 474-477, 2016.

[21] M. M. Butt, A. Mohamed, and M. Guizani, "On the effect of proportional fairness in energy transfer for wireless powered communication networks," in Proceedings of the 11th International Wireless Communications and Mobile Computing Conference (IWCMC '15), pp. 436-441, IEEE, Dubrovnik, Croatia, August 2015.

[22] H. Lee, K. Lee, H. Kim, B. Clerckx, and I. Lee, "Resource allocation techniques for wireless powered communication networks with energy storage constraint," IEEE Transactions on Wireless Communications, vol. 15, no. 4, pp. 2619-2628, 2016.

[23] Y. Dong, H. Zhang, M. J. Hossain, J. Cheng, and V. C. M. Leung, "Energy efficient resource allocation for OFDMA full duplex distributed antenna systems with energy recycling," in Proceedings of the IEEE Global Communications Conference (GLOBECOM '15), pp. 1-6, San Diego, Calif, USA, December 2015.

[24] D. Niyato, P. Wang, and D. I. Kim, "Admission control policy for wireless networks with RF energy transfer," in Proceedings of the 1st IEEE International Conference on Communications (ICC '14), pp. 1118-1123, Sydney, Australia, June 2014.
[25] M. Maso, C.-F. Liu, C.-H. Lee, T. Q. S. Quek, and L. S. Cardoso, "Energy-recycling full-duplex radios for next-generation networks," IEEE Journal on Selected Areas in Communications, vol. 33, no. 12, pp. 2948-2962, 2015.

[26] L. Xie, Y. Shi, Y. T. Hou, W. Lou, H. D. Sherali, and S. F. Midkiff, "Multi-node wireless energy charging in sensor networks," IEEE/ACM Transactions on Networking, vol. 23, no. 2, pp. 437450, 2015.

[27] F. Kelly, "Charging and rate control for elastic traffic," European Transactions on Telecommunications, vol. 8, no. 1, pp. 33-37, 1997, (GotoISI)://WOS:A1997WJ01500004.

[28] L. Li, M. Pal, and Y. R. Yang, "Proportional fairness in multirate wireless lans," in Proceedings of the 27th IEEE Conference on Computer Communications (INFOCOM '08), Phoenix, Ariz, USA, April 2008.

[29] W. Li, S. Wang, Y. Cui et al., "AP association for proportional fairness in multirate WLANs," IEEE/ACM Transactions on Networking, vol. 22, no. 1, pp. 191-202, 2014.

[30] H. Z. Shi, R. V. Prasad, E. Onur, and I. G. M. M. Niemegeers, "Fairness in wireless networks: issues, measures and challenges," IEEE Communications Surveys and Tutorials, vol. 16, no. 1, pp. 5-24, 2014.

[31] R. Jain, D.-M. Chiu, and W. R. Hawe, "A quantitative measure of fairness and discrimination for resource allocation in shared computer system," Tech. Rep., Digital Equipment Corporation, 1984.

[32] A. M. Zungeru, L.-M. Ang, S. Prabaharan, and K. P. Seng, "Radio frequency energy harvesting and management for wireless sensor networks," in Green Mobile Devices and Networks: Energy Optimization and Scavenging Techniques, pp. 341-368, CRC Press, New York, NY, USA, 2012.

[33] S. Boyd and L. Vandenberghe, Convex Optimization, Cambridge University Press, 2004.

[34] Z. Hadzi-Velkov, I. Nikoloska, G. K. Karagiannidis, and T. Q. Duong, "Wireless networks with energy harvesting and power transfer: joint power and time allocation," IEEE Signal Processing Letters, vol. 23, no. 1, pp. 50-54, 2016. 

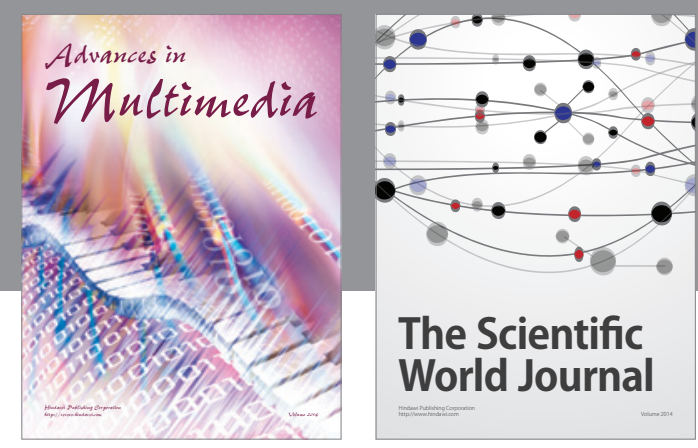

The Scientific World Journal
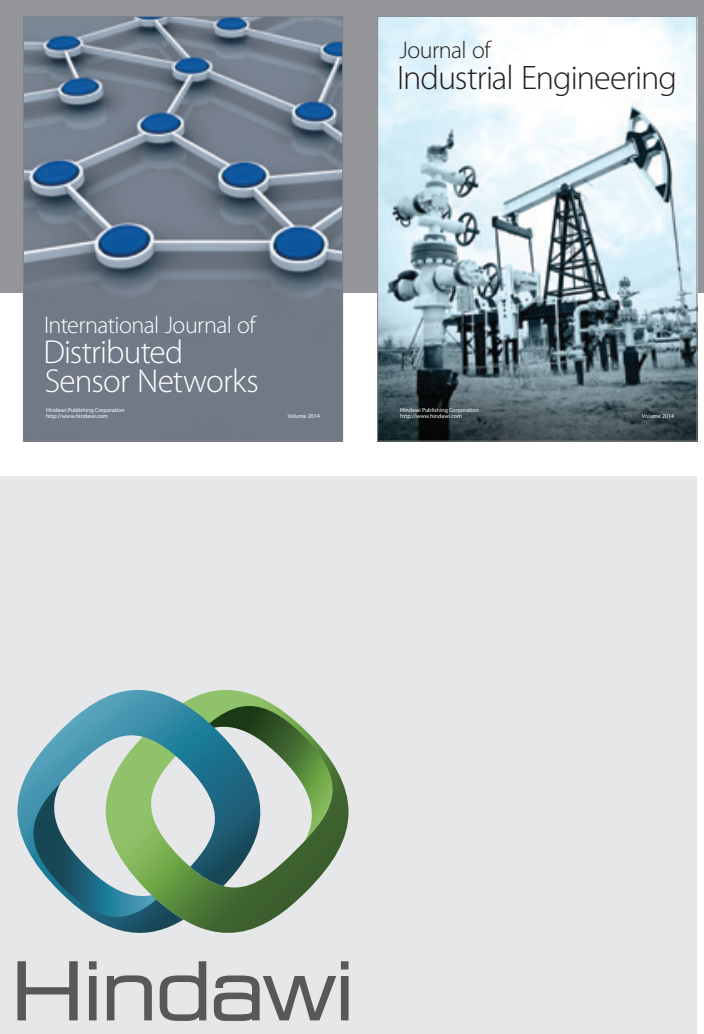

Submit your manuscripts at

http://www.hindawi.com

\section{Computer Networks} and Communications
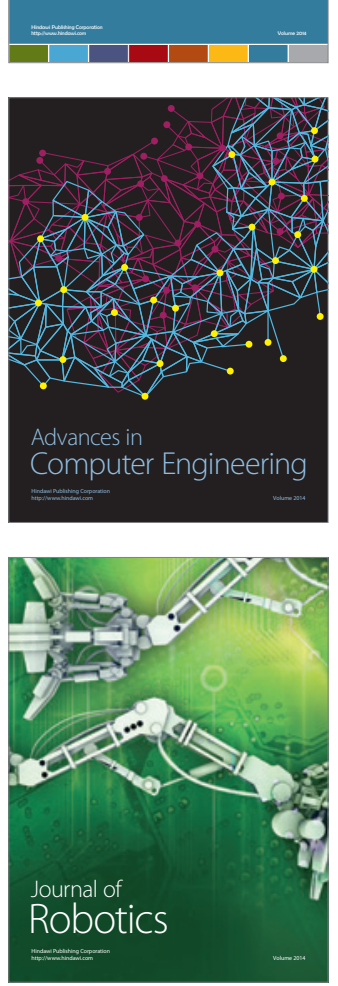
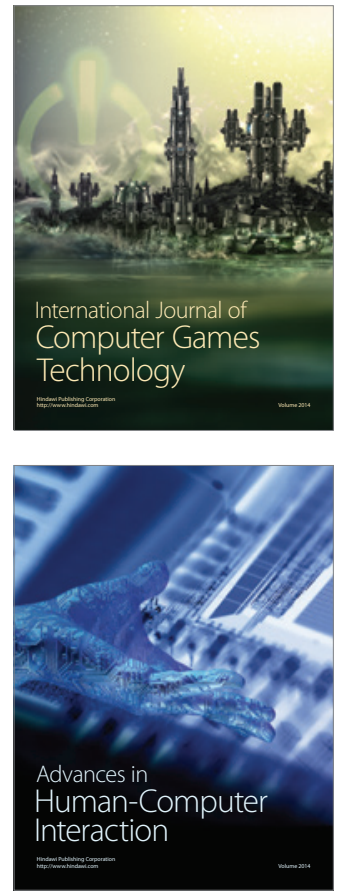
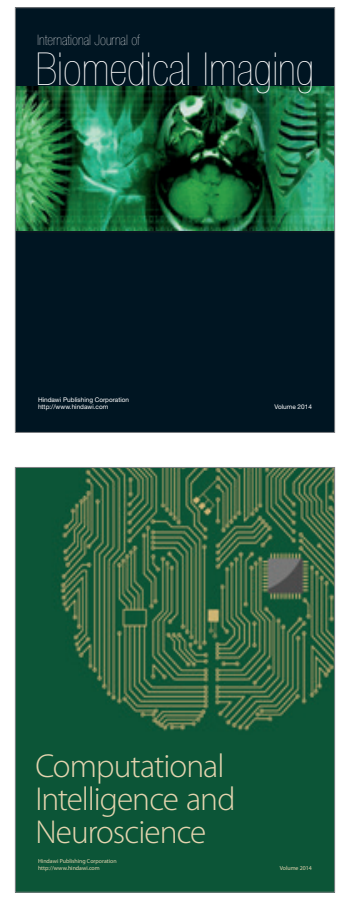
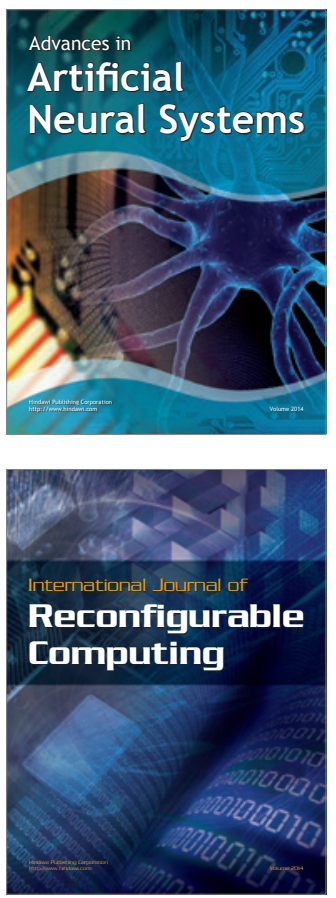
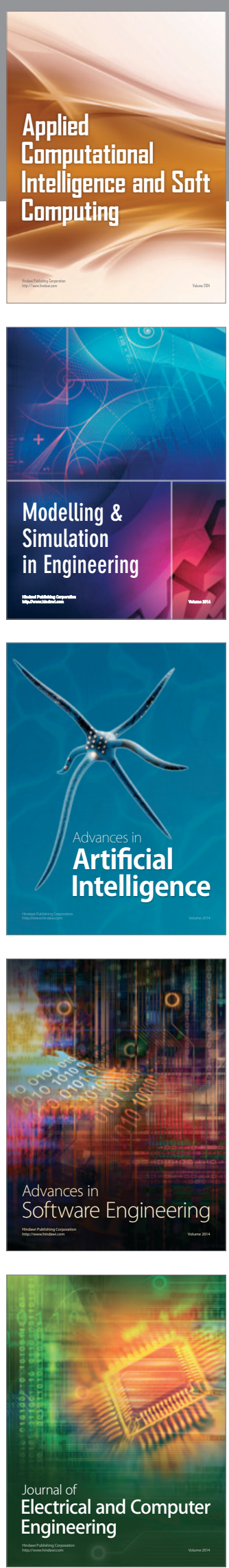